\title{
ELECTRICALLY COUPLED MEMS BANDPASS FILTERS
}

\author{
A Dissertation \\ Presented to \\ The Academic Faculty
}

By

\section{Siavash Pourkamali}

\author{
In Partial Fulfillment \\ Of the Requirements for the Degree \\ Master of Science in Electrical Engineering
}

Thesis Advisor: Professor Farrokh Ayazi

School of Electrical and Computer Engineering

Georgia Institute of Technology

April 2004 


\section{ELECTRICALLY COUPLED MEMS BANDPASS FILTERS}

Approved by:

Dr. Farrokh Ayazi, Advisor

Dr. Mark G. Allen

Dr. Oliver Brand

Date Approved: April 6, 2004 


\section{DEDICATION}

In the loving memory of my mother, Shahin Azimi 


\section{ACKNOWLEDGEMENTS}

I would like to express my deep appreciation to my thesis advisor and mentor, Professor Farrokh Ayazi for his support, encouragement, and advice. I would also like to thank the committee members, Professor Mark Allen and Professor Oliver Brand for their guidance and support. I would like to acknowledge my colleagues in the NMASP group, Reza Abdolvand, and Gavin Ho for their help and useful discussions in the research. Without their help, this work would not have been possible. I would also like to acknowledge all of my other colleagues in the IMEMS group for their assistance and friendship. I would like to thank the staff and students of the MiRC cleanroom at the Georgia Institute of Technology for all of their help with the equipment. Finally, I wish to thank my family, especially my father, my dear departed mother, my brother and sister for all of their support. 


\section{TABLE OF CONTENTS}

ACKNOWLEDGEMENTS $\ldots \ldots \ldots \ldots \ldots \ldots \ldots \ldots \ldots \ldots \ldots \ldots \ldots \ldots \ldots \ldots \ldots \ldots \ldots \ldots \ldots \ldots \ldots \ldots$ iii

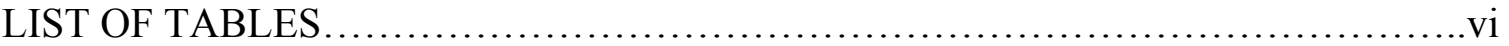

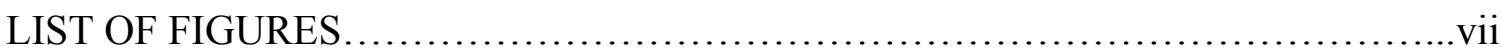

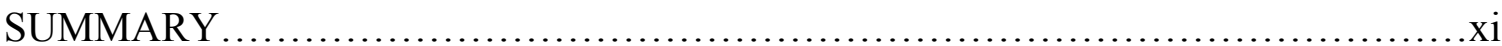

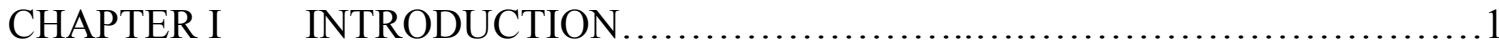

CHAPTER II $\quad$ BACKGROUND AND MOTIVATION ............................5

2.1. Capacitive Silicon MEM Resonators..................................6

2.1.1. All-Silicon Resonators using the HARPSS Technology ............7

2.1.2. Fully Single Crystal Silicon Resonators with Submicron DryEtched Capacitive Gaps .........................................9

2.2. Micromechanical Bandpass Filters................................ 10

2.2.1. Mechanically Coupled Bandpass Filters..........................11

CHAPTER III ELECTROMECHANICAL MODELING OF CAPACITIVE BEAM

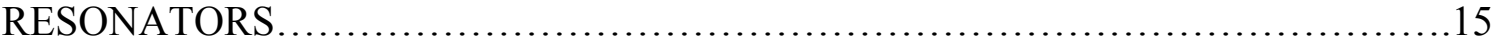

3.1. Equivalent Circuit Model for Two-Port Beam Resonators....................16

3.1.1. Electrostatic Tuning of Micromechanical Beam Resonators......26 
CHAPTER IV ELECTRICAL COUPLING OF MEM RESONATORS FOR

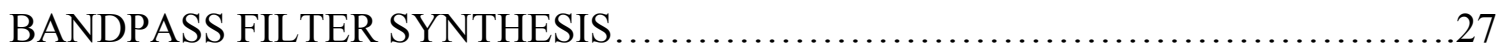

4.1. Active Coupling of Resonators...........................................27

4.2. Capacitive Coupling of Resonators...................................30

4.2.1. Insertion Loss in Capacitively Coupled Filters.....................36

4.2.2. Lossy Coupling Capacitors........................................37

4.3. Electrostatic Coupling of Resonators ..................................38

4.3.1. Electrostatic Coupling Force......................................39

4.3.2. Electromechanical Modeling..................................41

CHAPTER V PRACTICAL DEMONSTRATION OF ELECTRICALLY COUPLED MICROMECHANICAL FILTERS..................................47

5.1. Electrically Cascaded Filters..........................................4 47

5.2. Discrete Capacitivly Coupled Filters..................................52

5.3. Capacitively Coupled Filters with Integrated Coupling Capacitors............54

5.4. Electrostatically Coupled Filters......................................60

CHAPTER VI CONCLUSION AND FUTURE DIRECTIONS...................66

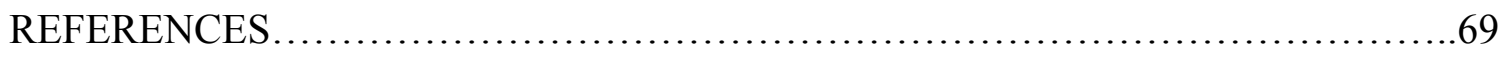




\section{LIST OF TABLES}

Table 5.1. Calculated and measured overall and individual Q's for cascaded

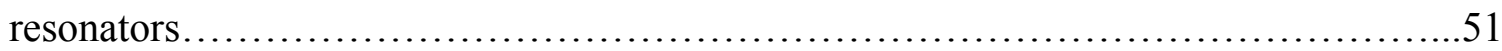

Table 5.2. Calculated resonator parameters and filter bandwidth with different polarization voltages and the measured values................................60

Table 5.3. Calculated resonator and coupling parameters used in PSPICE simulations of electrostatically coupled filter of Fig. 5.12 and comparison of simulated/calculated resonance peak separation with the measured values for different polarization voltages 


\section{LIST OF FIGURES}

Figure 2.1. SEM view of a $500 \mu \mathrm{m}$ long, $5 \mu \mathrm{m}$ wide, $20 \mu \mathrm{m}$ thick HARPSS SCS clampedclamped beam resonator with 700nm capacitive transducer gaps, and close-up of its electrode area................................................................. 8

Figure 2.2. SEM view of a $180 \mu \mathrm{m}$ long, $4 \mu \mathrm{m}$ wide, $4.5 \mu \mathrm{m}$ thick fully SCS clampedclamped beam resonator with 350nm dry-etched capacitive transducer gaps, and close-up of its electrode area...........................................................

Figure 2.3. Schematic diagram of a mechanically coupled filter array comprised of beam resonators coupled with compliant coupling elements.....

Figure 2.4. Schematic diagram of a second order mechanically coupled system, comprised of two identical resonators and one coupling element....

Figure 2.5. Schematic diagram of a third order mechanically coupled system, comprised of three identical resonators. 13

Figure 3.1. Operating configuration of a two-port HARPSS clamped-clamped capacitive beam resonator .16

Figure 3.2. Admittance parameters for a two-port network

Figure 3.3. Mode shape for the first flexural resonance mode of a clamped-clamped beam resonator. $\mathrm{Y}(\mathrm{x})$ is the normalized displacement function along the beam length having a value of one in the middle. .20

Figure 3.4. Small-signal equivalent circuit for a two- port micromechanical resonator...24

Figure 3.5. Alternative way of demonstration of the equivalent small signal model for a two-port micromechanical resonator.

.25 
Figure 4.1. Schematic diagram of an electrically cascaded resonant system comprised of micromechanical resonators interfaced with active electrical components in between...27

Figure 4.2. Frequency response of a typical bandpass filter and definition of the shape factor. .28

Figure 4.3. (a) Simulation results for cascaded resonators with individual $Q=10,000$ $(600 \mathrm{kHz}) .(\mathrm{b})$ Simulation results of cascaded resonators with identical overall quality factors $\&$ different

orders .29

Figure 4.4. Schematic diagram of a capacitively-coupled microelectromechanical filter.31 Figure 4.5. Electrical schematic diagram of a second order capacitively coupled resonant system and its frequency response. .32

Figure 4.6. Electrical schematic diagram of a third order coupled resonant system and its frequency response. .34

Figure 4.7. Schematic diagram of a closed loop array of capacitively-coupled microelectromechanical resonators .35

Figure 4.8. Frequency response of a typical third order capacitively coupled filter before and after adding terminating resistors .35

Figure 4.9. Simulation results for $600 \mathrm{kHz}$ capacitively-coupled $2^{\text {nd }} \& 3^{\text {rd }}$ order filters. A higher resonator $\mathrm{Q}$ results in a lower insertion loss. Coupling cap: $\mathrm{C}_{\mathrm{c}}=0.15 \mathrm{pF}$ ( $2^{\text {nd }}$ order); $\& \mathrm{C}_{\mathrm{c}}=0.2 \mathrm{pF}\left(3^{\text {rd }}\right.$ order $)$

Figure 4.10. Effect of the finite Q of the coupling capacitors on the frequency response of a third order capacitively coupled filter. .38 
Figure 4.11. Schematic diagram of an electrostatically coupled beam filter array....

Figure 4.12. Schematic diagram of two electrostatically coupled clamped-clamped beam resonators.

Figure 4.13. Equivalent lumped element model of a second order electrostatically coupled filter and its frequency response.

Figure 4.14. Electrical Equivalent circuit of a second order electrostatically coupled filter .46

Figure 5.1. A $300 \mu \mathrm{m}$ long, $6.5 \mu \mathrm{m}$ wide, $20 \mu \mathrm{m}$ tall clamped-clamped beam HARPSS SCS resonator with a gap spacing o700nm .48

Figure 5.2. Schematic diagram of the test setup used for implementation of second and third order electrically cascaded micromechanical filters

Figure 5.3. Measured frequency response of cascaded resonators demonstrating Q amplification and shape factor improvement. .50

Figure 5.4. Frequency response of $2^{\text {nd }}$ and $3^{\text {rd }}$ order bandpass filters achieved by active electrical cascading of HARPSS resonators. .51 Figure 5.5. Schematic of the test setup for arrays of capacitively coupled SCS beam resonators. .52

Figure 5.6. Frequency response of capacitively coupled resonators with off-chip interconnections a) measured frequency spectrum; b) PSPICE Simulation result. .53

Figure 5.7. Frequency response of the capacitively coupled resonators after adjusting the polarization voltages to increase the bandwidth a) measured frequency response; b) PSPICE simulation result.................................................54 
Figure 5.8. SEM view of a second order capacitively coupled filter with integrated

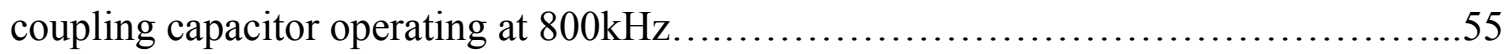

Figure 5.9. Schematic of the test setup for measurement of arrays of capacitively coupled SCS beam resonators with integrated coupling capacitor. .56

Figure 5.10. Frequency response of the $800 \mathrm{kHz}$ capacitively coupled filter with integrated coupling capacitor. Filter bandwidth changes due to the applied polarization voltages

Figure 5.11. PSPICE simulation results for the capacitively coupled filter of Fig. 5.8 with polarization voltage values similar to the measurement conditions of Fig. 5.10. .59

Figure 5.12. SEM of a fabricated second order clamped-clamped electrostatically coupled beam filter. .60

Figure 5.13. Frequency response of the electrostatically coupled filter of Fig. 5.12 with different sets of polarization voltages applied to the beams.

Figure 5.14. PSPICE simulation results for the electrostatically coupled filter of Fig. 5.12 with polarization voltage values similar to the measurement situation in Fig. 5.13......63 Figure 5.15. Resonance peak separation versus the square of polarization voltage difference $\left(\Delta \mathrm{V}_{\mathrm{p}}{ }^{2}\right)$ for the electrostatically coupled filter of Fig. $5.12 \ldots \ldots \ldots \ldots \ldots \ldots \ldots \ldots 6$ Figure 6.1. Schematic diagram of parallel actively coupled resonator arrays for implementation of wide bandwidth, highly selective MEM filters. .68 


\section{SUMMARY}

This dissertation reports, for the first time, on the electrical coupling of microelectromechanical (MEM) resonators for high order bandpass filter synthesis. Electrical coupling of MEM resonators has a strong potential for extension of the operating frequency of MEM bandpass filters into the ultra high frequency (UHF) range and provides higher tunability and design flexibility compared to the mechanical coupling approach. Various schemes of electrical coupling are presented in this dissertation. Electromechanical models of clamped-clamped beam resonators, and various types of electrically coupled filters are presented. Lower frequency prototypes of electrically coupled filters with operating frequencies in the hundreds of $\mathrm{kHz}$ are implemented using micromechanical single crystal silicon clamped-clamped beam resonators. Measurement results are in good agreement with the developed electrical equivalent models of the filters. It is demonstrated that the characteristics of electrically coupled filters can be widely tuned by changing the DC polarization voltages. 


\section{CHAPTER I}

\section{INTRODUCTION}

Increasing demand for miniaturized electrical appliances especially wireless communication systems with higher performance and lower cost has emerged MEM (Micro-Electro-Mechanical) resonators as potential candidates for use in various RF frequency-selection as well as sensory applications. Such devices combine the attractive features of small size, low cost batch fabrication, potential for CMOS compatibility and electrical tunability. A great amount of research is currently underway to replace offchip, bulky frequency selective components in RF electronics systems with integrated microresonators.

Development of silicon micromachining technologies during the past few decades has facilitated fabrication and characterization of a variety of polysilicon and single crystal silicon resonators with dimensions in the low micron range. Higher quality factor provided by silicon, compatibility with IC integration, better long term and temperature stability and finally availability of a variety of batch-fabrication processes with micromachining techniques has made silicon an attractive structural material for microresonators. A variety of high quality factor $(\mathrm{Q})$ microresonators with polysilicon [1-6] or single-crystal silicon (SCS) [7-11] as the resonating element have been reported so far.

From a filter design point of view, a single microresonator, although having a high quality factor in the order of a few thousands, is a first order bandpass filter 
providing limited frequency selectivity (shape factor). Therefore higher order bandpass filters consisting of a number of coupled resonators are required in order to achieve the desired selectivity for most of the applications.

On the resonator coupling front, mechanical coupling technique, traditionally used for implementation of high order filters from individual mechanical resonators [12], has been applied to MEM resonators for filter synthesis [13-17]. Electrically sensed and actuated MEM filters up to the third order [14] and center frequencies up to 68MHz [15] as well as electrically actuated and optically sensed filters up to the $20^{\text {th }}$ order at center frequencies of a few $\mathrm{MHz}$ [17] have been reported using the mechanical coupling technique. However, in the UHF frequency range $(0.3-3 \mathrm{GHz})$ and above, which is the band of interest for many wireless applications, due to the very small size of the resonator element $(<10 \mu \mathrm{m})$, mechanical coupling will require sub-micron in size coupling elements (i.e., wires) that are difficult to fabricate using optical lithography. In addition, filter characteristics are sharply dependant on the positioning and dimensions of the coupling elements [18] and optimized design of a filter will require mechanical design expertise and specialized simulation tools.

This dissertation reports, for the first time, a variety of novel filter synthesis approaches based on the electrical coupling of individual SCS resonators: 1. active cascading; 2. capacitive coupling; and 3. electrostatic coupling.

The main advantage of electrical coupling approaches in general is their greater potential for extension of the operating frequency into the UHF range. Filter synthesis using electrical coupling does not require mechanical design expertise and provides more design flexibility for electrical engineers and higher tunability/programmability after the 
device is fabricated. Passive electrical coupling of microresonators using shunt capacitors (capacitive coupling) has a greater potential for extension into the ultra high frequency (UHF) range, and provides superior tunability and design flexibility compared to the mechanical coupling technique. The electrostatic coupling technique does not require any distinct coupling component and compared to the other electrical and mechanical coupling techniques provides the highest tunability and design flexibility and alleviates some of the problems associated with capacitive coupling (such as DC biasing of the coupling capacitor).

The contributions of this dissertation are the following:

1) Introduction of the concept of electrical coupling in capacitive two-port MEM resonators.

2) Electromechanical modeling and simulation of arrays of electrically coupled bandpass filters.

3) Implementation of on-chip low frequency prototypes of electrically coupled MEM bandpass filters and test and characterization of such devices.

This dissertation is organized in six chapters. A brief outline of the dissertation is presented below.

Chapter 1 introduces this dissertation and discusses the contributions of the presented research work.

Chapter 2 discusses the background to the micromechanical resonators and mechanically coupled filters. 
Chapter 3 presents electromechanical modeling of two-port clamped-clamped beam resonators. A device concept and equivalent circuit model for two-port resonators are presented in this section.

Chapter 4 discusses the theory and electromechanical modeling of the various electrical coupling approaches presented in this work. Simulation results are provided to support the theory where required.

Chapter 5 presents practical implementation and measurement results of low frequency prototypes of electrically coupled MEM bandpass filters. The theoretical models are validated by comparison to the test results.

Chapter 6 briefly summarizes this research and gives recommendations for frequency and performance enhancement of electrically coupled filter arrays. 


\section{CHAPTER II}

\section{BACKGROUND AND MOTIVATION}

A microelectromechanical resonator is a micromachined mechanical structure with integrated transducers that can be excited to vibrate at one or more of its resonance modes. A classic example of a mechanical resonator in macro domain is a guitar string. Like guitar strings, micromechanical beam resonators can operate in a wide range of frequencies for different structural dimensions.

The demonstration of MEM resonators dates back to 1967 with the invention of the resonant gate transistor (RGT) at Westinghouse Research Laboratories [19]. The dimension of this gold beam was $0.1 \mathrm{~mm}$ in length, and $5-10 \mu \mathrm{m}$ in thickness, resonating at $5 \mathrm{kHz}$ with a quality factor of 500 .

A number of different materials with specific characteristics have been used as the resonating element for mechanical resonator. Piezoelectric materials such as quartz or lead-zirconate-titanates (PZT), were also used to implement piezoelectric resonators [12]. However, all of these materials lack the capability of integration with electronics. On the other hand, piezoelectric actuation and detection requires the drive and sense electrodes to be in physical contact with the resonating body, causing excessive loss and quality factor degradation for such resonators.

Currently, quartz crystal is one of the best choices available choices as the resonator material. The history of quartz can be dated back to over fifty years age [20]. Since then, the performance of quartz frequency standards have gradually improved with new innovations such as use of temperature-compensated crystal, discovery of SC-cut 
crystal [21], and invention of SAW resonators and filters. These new discoveries have lead to low-cost, extremely stable and precise quartz crystals with high mechanical quality factors (Q). However, like other piezoelectric resonators, the biggest challenge that quartz resonators face even until this day is that their batch processing is not compatible with IC fabrication technology.

\subsection{Capacitive Silicon MEM Resonators}

Lately, silicon resonators (both poly and single crystalline) have attracted much attention due to well-established processing technology available in IC industry and compatibility with IC integration. In addition, capacitive actuation and sensing has been under investigation as a superior transduction mechanism for high frequency micromechanical resonators. Capacitive signal transduction is performed without the need for direct physical contact between the electrodes and the resonating body, resulting in a higher potential quality factor for the resonator. In addition, any conductive material can be used as the capacitive actuator avoiding the need for IC-incompatible piezoelectric materials in the structure of the device, causing potentially higher long-term and temperature stability.

Various types of poly and single crystal silicon resonators have been reported so far in the literature. Polysilicon surface micromachining technology and its variations have been deployed for fabrication of high quality factor polysilicon capacitive resonators with polysilicon [1-3] or metal [4-6] electrodes and self-aligned deepsubmicron capacitive transducer gaps. As the resonators are shrunk down in size to reach high operating frequencies in the VHF $(30 \mathrm{MHz}-300 \mathrm{MHz})$ and UHF $(300 \mathrm{MHz}-3 \mathrm{GHz})$ 
range, large electrostatic actuation forces will be required to resonate their ultra-stiff structures. On the other hand having large enough sensitivity to sense the small vibration amplitudes of such structures calls for electrostatic actuation and sense gap sizes in the deep submicron (below 100nm) range. The capacitive transduction gaps of the surface micromachined polysilicon resonators are defined in a self-aligned manner by the thickness of a deposited sacrificial oxide layer and can be reduced down to the required levels.

Single-crystal silicon (SCS) is a more attractive material for micromechanical resonators due to its inherent higher mechanical quality factor [22], low defect densities, and independence from different process parameters. However since SCS can not be deposited using conventional CVD techniques, fabrication of SCS resonators with ultrathin capacitive transducer gaps is not a trivial task. Most of the SCS resonators reported so far in the literature have either complex non-capacitive drive and sense mechanisms [23] or large capacitive gaps $[10,11]$. All silicon resonators with SCS resonating element and self-aligned ultra-thin capacitive gaps have been demonstrated using the HARPSS fabrication technology $[24,25]$.

\subsubsection{All-Silicon Resonators using the HARPSS Technology}

The HARPSS process [24,25], is a mixed bulk/surface fabrication technology that is capable of simultaneously producing $10-100$ 's of microns thick, electrically isolated, poly and single-crystal silicon microstructures, with various size capacitive gaps ranging from deep sub-micron to tens of microns. The HARPSS process has been utilized to fabricate all-silicon microresonators with SCS as the resonating element and polysilicon actuation and sense electrodes [7-9]. Similar to surface micromachining technologies, 
the capacitive gap size of the HARPSS devices are defined by the thickness of a deposited sacrificial oxide layer and can be reduced to ultra small values as required for high frequency operation. In addition, unlike the surface micromachined resonators with thickness limited to 1-3microns, HARPSS resonators can be as thick as a few tens of microns, providing a much larger transduction area. Higher signal transduction area in turn results in higher power handling capability and signal to noise ratio.

Figure 2.1 shows the SEM view of a typical HARPSS SCS clamped-clamped beam resonator fabricated on a regular silicon substrate and close-up of its electrode area showing the submicron capacitive gap in between the SCS resonator and polysilicon electrodes. HARPSS SCS beam resonators [26] are used in this work for practical demonstration of active coupling and discrete capacitive coupling as explained later.
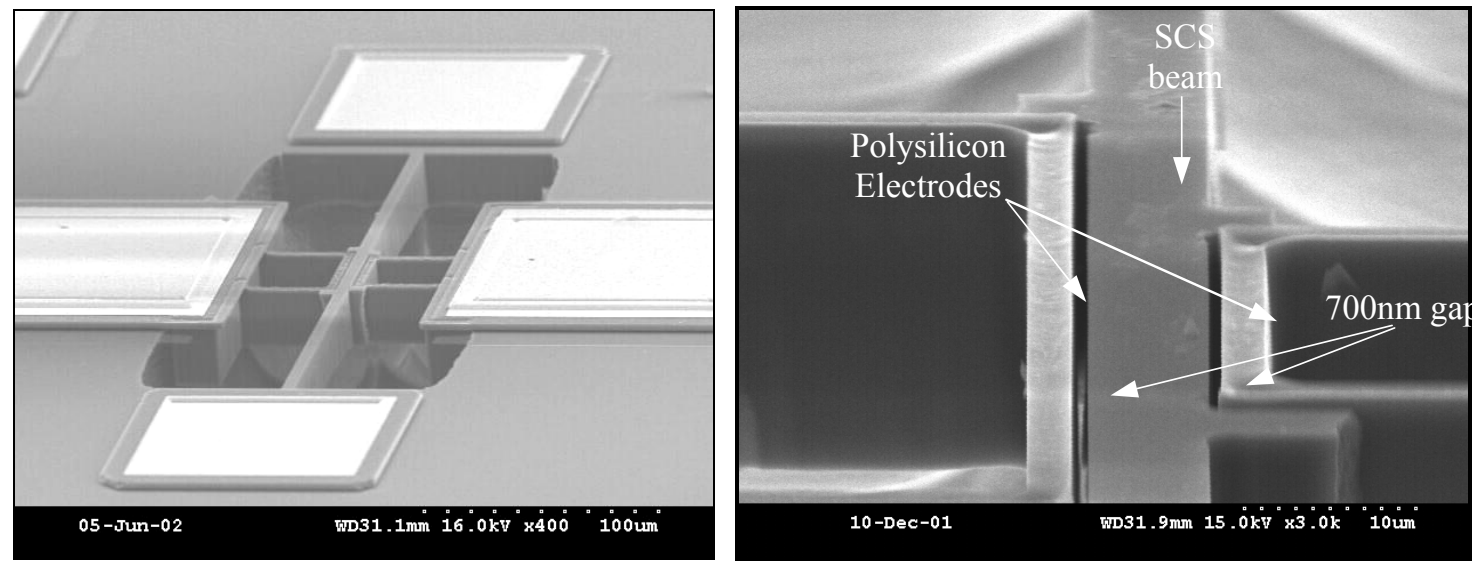

Figure 2.1. SEM view of a $500 \mu \mathrm{m}$ long, $5 \mu \mathrm{m}$ wide, $20 \mu \mathrm{m}$ thick HARPSS SCS clampedclamped beam resonator with $700 \mathrm{~nm}$ capacitive transducer gaps, and close-up of its electrode area. 


\subsubsection{Fully Single Crystal Silicon Resonators with Submicron Dry-Etched Capacitive Gaps}

The second type of SCS resonators with submicron capacitive gaps recently developed in our group at Georgia Tech. are the fully SCS resonators with submicron dry-etched capacitive gaps [27]. Such resonators are fabricated on low resistivity siliconon-insulator (SOI) substrates and have SCS resonating structure and transducer electrodes. The deep-submicron high aspect-ratio transducer gaps are created through a novel processing technique that uses low-cost micron-resolution optical lithography. The mask features defining the width of the transducer gaps are created in a self-aligned manner by the thickness of a deposited sacrificial polysilicon layer, and the submicron capacitive gaps are dry-etched using the Bosch process.

Figure 2.2 shows the SEM view of a fabricated fully SCS clamped-clamped beam resonator and its close-up of the electrode area, showing the 350nm capacitive gap in between the SCS electrodes and the SCS beam.

The all SCS capacitive resonators have been used in this work for implementation of capacitively coupled filters with integrated coupling capacitors. 

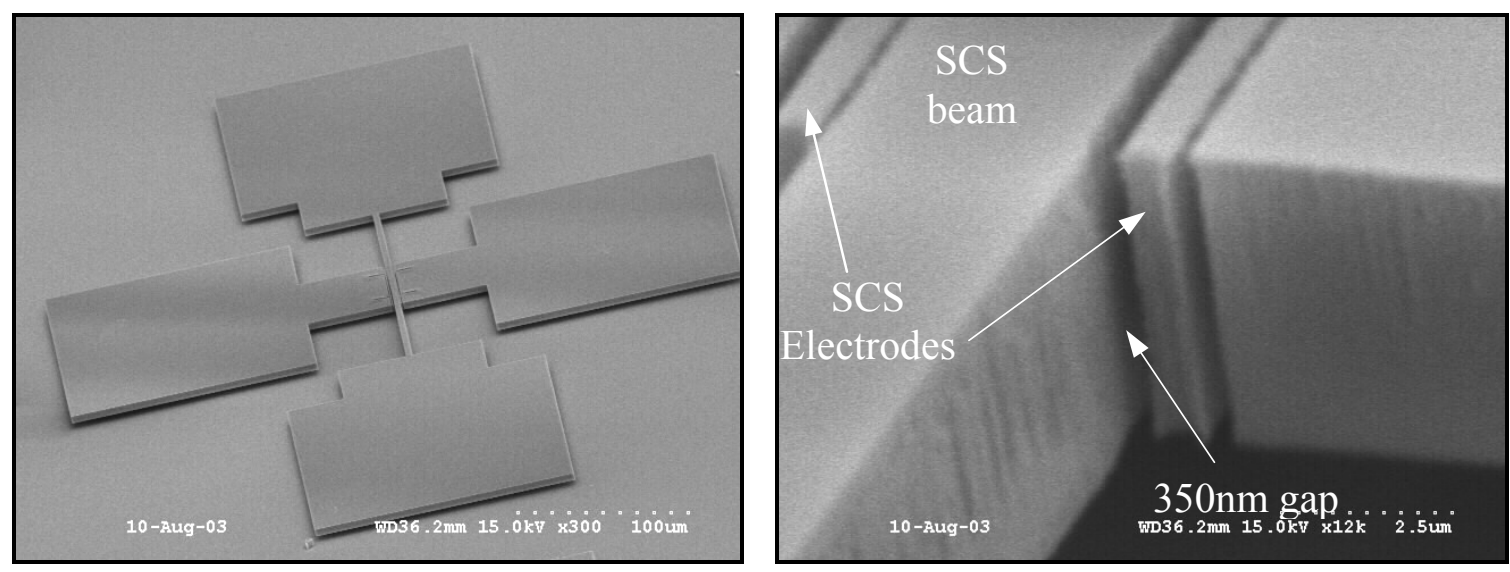

Figure 2.2. SEM view of a $180 \mu \mathrm{m}$ long, $4 \mu \mathrm{m}$ wide, $4.5 \mu \mathrm{m}$ thick fully SCS clampedclamped beam resonator with 350nm dry-etched capacitive transducer gaps, and close-up of its electrode area.

\subsection{Micromechanical Bandpass Filters}

Mechanical filters are used in systems that demand narrow bandwidth, low loss and good stability [12]. Because of the high Q and excellent temperature and aging characteristics these requirements can be achieved with mechanical filters.

From an electrical filter design point of view, a single microresonators although having a high quality factor in the order of a few tens of thousands, is a first order bandpass filter providing limited frequency selectivity (shape factor). Therefore higher order bandpass filters consisting of a number of coupled resonators are required to achieve the desired selectivity for most of the applications. High quality factor of mechanical resonators allows realization of mechanical filters with narrow bandwidth as small as $0.1 \%$ and smaller without introducing excessive loss or passband rounding.

Development of mechanical filters for use in electronic systems dates back to the 1920s. Campbell and Wagner developed the first mechanical filter in 1915 [28]. Nowadays mechanical filters have become a vital part of the electronics industry and 
several types of mechanical filters using different types of resonators, including metal alloy, PZT-ceramic [29] and zinc oxide [30] resonators, with different transduction and mechanical coupling mechanisms have been developed so far.

Development of the first micromechanical resonator in 1967 [19], provided new opportunities for miniaturization of the mechanical filters. Mechanical coupling technique has been applied to micromechanical resonators for filter synthesis [13-17]. However, mechanical coupling for ultra-small micromechanical resonators with operating frequencies in the in the VHF $(30-300 \mathrm{MHz})$ and UHF $(0.3-3 \mathrm{GHz})$ frequency range, which is the band of interest for most of the modern wireless systems, faces issues and challenges that can not be easily overcome by means of the available commercialized technologies.

\subsubsection{Mechanically Coupled Bandpass Filters}

According to the vibration theory [31], a mechanical system with $\mathrm{n}$ degrees of freedom has $\mathrm{n}$ resonance modes. Based on the same concept, if a number of micromechanical resonators are physically connected to each other with compliant coupling elements (as shown in Fig 2.3), the resulting structure will have different resonance modes and the number of modes is equal to the number of coupled resonators. 


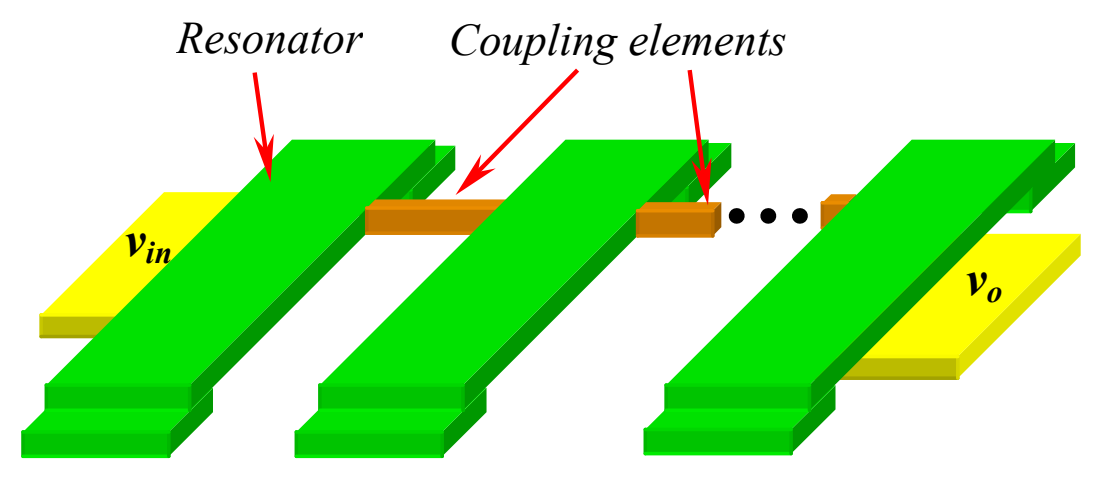

Figure 2.3. Schematic diagram of a mechanically coupled filter array comprised of beam resonators coupled with compliant coupling elements.

When the coupled resonators have equal center frequencies, the first resonance mode of the coupled array will have the same natural frequency as the individual resonators and the rest of the resonance modes occur at close but slightly higher frequencies. The frequency difference between the resonance modes is determined by the ratio of the stiffness of the resonator to the stiffness of the coupling element. More compliant coupling elements result in smaller separation of the resonance modes.

As a simple example lets assume the mechanical system depicted in Fig. 2.4. Two similar resonators, each consisting of a mass and a clamped-free beam as the stiffness element are coupled with a spring. In the first resonance mode the two resonators resonate in phase and since the coupling spring is not deformed, it has no effect on the resonance of the resonators and the resonance frequency will be the same as the resonance frequency of a single resonator (assuming negligible mass of the coupling element). In the second resonance mode the two resonators resonate with $180^{\circ}$ phase shift and the coupling spring enters the game. The equivalent stiffness of each of the resonators increases by twice the stiffness of the coupling spring and causes a shift in the resonance frequency as shown in Fig. 2.4. 

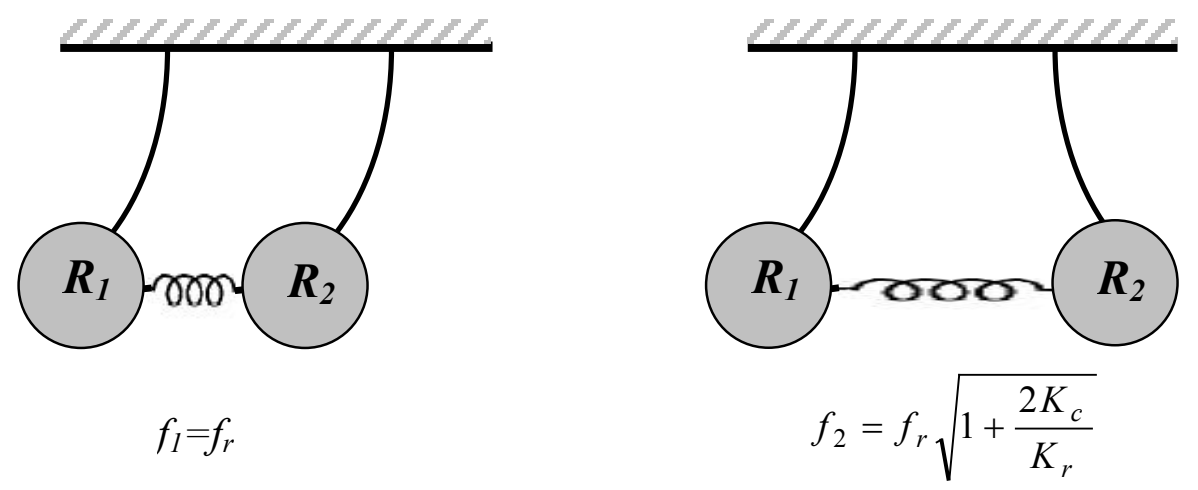

$f_{r}=$ resonator's resonance frequency

$K_{r}=$ Resonator's stiffness

$K_{c}=$ Stiffness of the coupling element

$B W \approx f_{2}-f_{1} \approx-\frac{f_{r} K_{c}}{K_{r}}$

Figure 2.4. Schematic diagram of a second order mechanically coupled resonant system, comprised of two identical resonators and one coupling element.

For a third order system (Fig. 2.5), the case is more complicated.
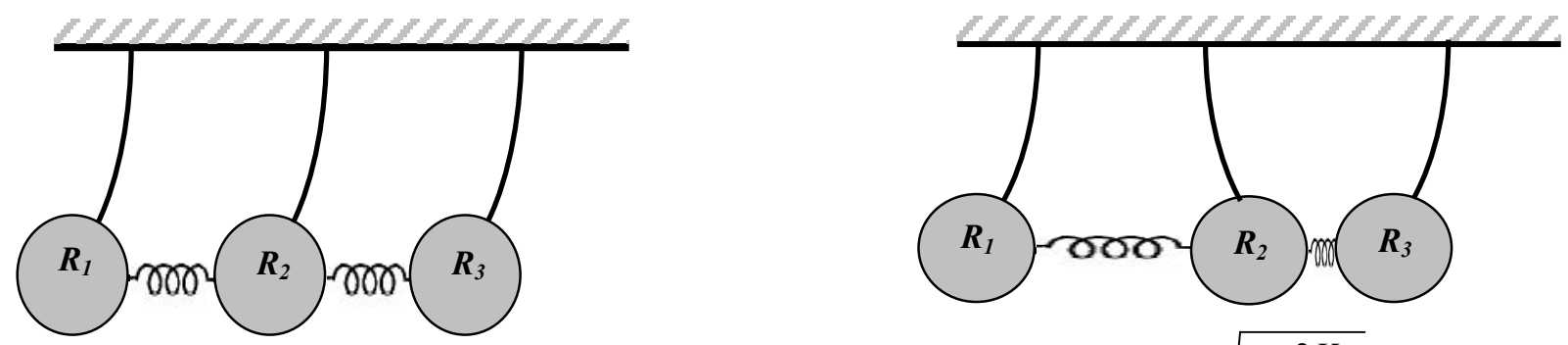

$f_{1}=f_{r}$

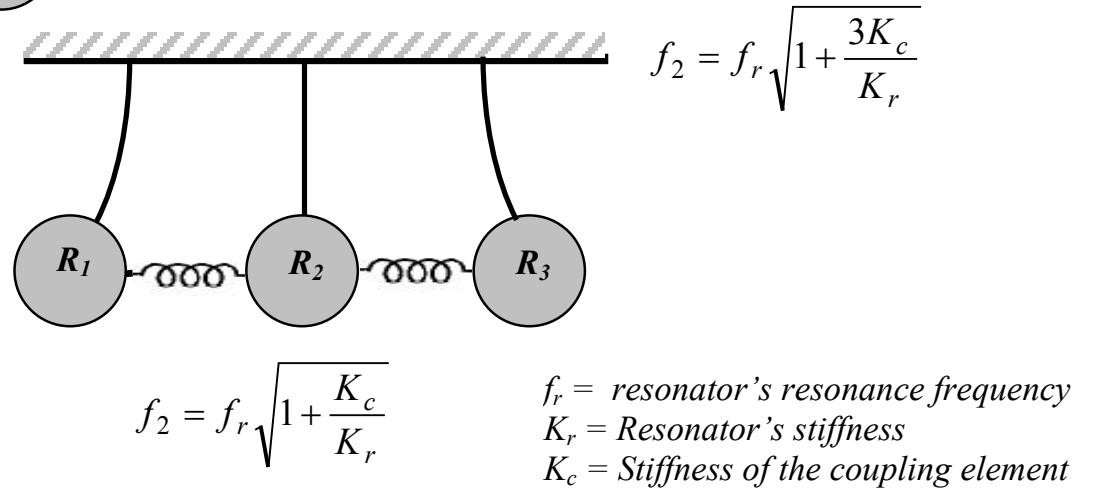

Figure 2.5. Schematic diagram of a third order mechanically coupled system, comprised of three identical resonators and two coupling elements. 
Having several resonance modes with close natural frequencies provides sharper transition from passband to the stopband, making the filter characteristic closer to an ideal filter.

Electrically sensed and actuated mechanically coupled MEM filters up to the third order [14] and center frequencies up to $68 \mathrm{MHz}$ [15] have been implemented using surface micromachined polysilicon resonators. Mechanically coupled MEM filters up to the $20^{\text {th }}$ order with electrical actuation and optical sensing schemes have been demonstrated at center frequencies of a few $\mathrm{MHz}$ [17]. The major bottlenecks for mechanically coupled micromechanical filters are: 1 . The need for ultra-compliant and ultra-light (to avoid mass loading of the resonators) coupling elements for narrow bandwidth filters with small dimensions, which can hardly be implemented using today's available lithography tools, 2 . Tolerances in size and position of the coupling element will transfer directly to the filter characteristics and no tuning capability is available for the coupling strength, in other words filter characteristics can not be tuned after fabrication. 


\section{CHAPTER III}

\section{ELECTROMECHANICAL MODELING OF CAPACITIVE BEAM RESONATORS}

In order to have a basic understanding of the electrically coupled arrays of resonators and analyze such devices using the electrical network theory and circuit simulation softwares, derivation of an electrical equivalent circuit for the individual resonators is necessary.

The schematic diagram of a typical electrostatically operated two-port clampedclamped beam resonator is shown in Fig. 3.1. The resonators used in this work have a two-port configuration with separate drive and sense electrode. To drive these microstructures into resonance, an $\mathrm{AC}$ voltage is applied to the drive electrode, and a DC voltage known as the polarization voltage is applied to the resonating body, resulting in an electrostatic force between the electrode and the beam that consequently causes the motion of the resonator. The capacitance change between the sense electrode and the beam due to the movement of the beam, generates an electrical current in the sense electrode. The microstructure motion is detected by sensing the current at the sense electrode of the device. When the frequency of the applied drive voltage is equal to the mechanical natural frequency of the resonator, motion of the microstructure and consequently the output current of the device reach their maximum values. The amplitude of vibration at the resonance frequency is $Q$ times $(\mathrm{Q}=$ quality factor $)$ times larger than the DC displacement amplitudes with the same drive voltage value. 


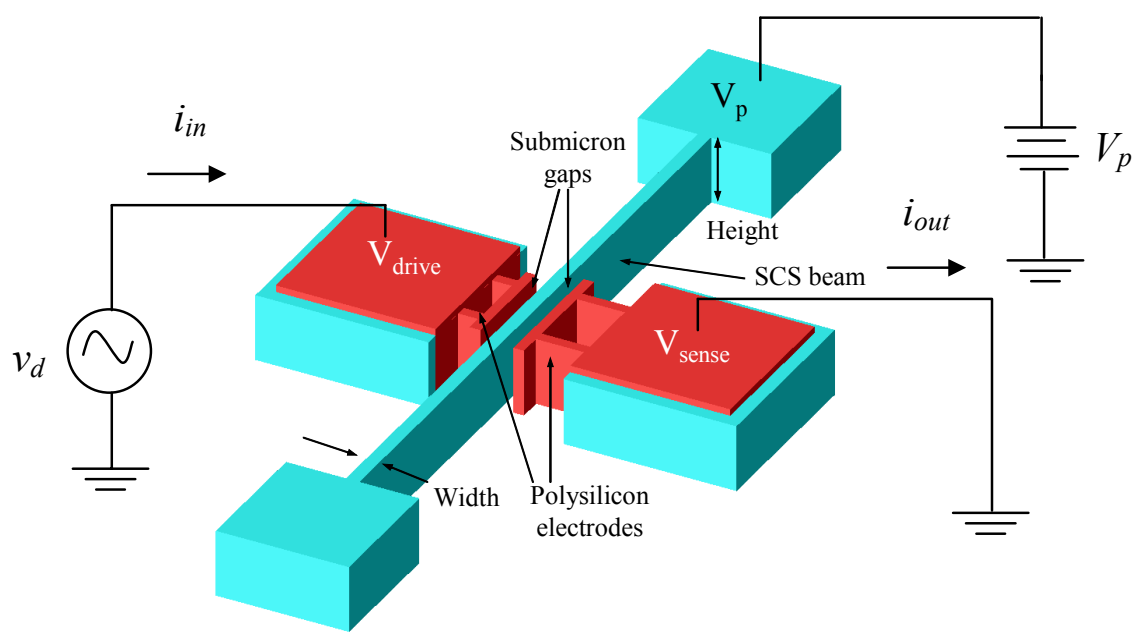

Figure 3.1. Operating configuration of a two-port HARPSS clamped-clamped capacitive beam resonator.

\subsection{Equivalent Circuit Model for Two-Port Beam Resonators}

In the electrical domain the two-port resonators can be treated as a two-port network fully characterized by four network model parameters. The network parameters can be admittance $(\mathrm{y})$, impedance $(\mathrm{z})$, hybrid $(\mathrm{h})$, transmission $(\mathrm{T})$ parameters or scattering (S) parameters. The admittance parameters are the easiest to derive analytically in this case and can be derived by calculation of short circuit currents for the resonators.

A general two-port network and the definition of its admittance parameters are shown in Fig. 3.2. 


$$
\begin{gathered}
Y=\left[\begin{array}{ll}
y_{11} & y_{12} \\
y_{21} & y_{22}
\end{array}\right] \stackrel{i_{\text {out }}}{=}+ \\
\left.y_{11}=\left(\frac{i_{\text {in }}}{v_{\text {in }}}\right)_{v_{\text {out }}=0} y_{12}=\left(\frac{i_{\text {in }}}{i_{\text {out }}=y_{11}}\right)_{\text {out }}\right)_{v_{\text {in }}=0} v_{\text {in }}+y_{22} v_{\text {out }} \\
y_{21}=\left(\frac{i_{\text {out }}}{v_{\text {in }}}\right)_{v_{\text {out }}=0}^{i_{\text {in }}} y_{22}=\left(\frac{i_{\text {out }}}{v_{\text {out }}}\right)_{v_{\text {in }}=0}
\end{gathered}
$$

Figure 3.2. Admittance parameters for a two-port network.

In a two-port resonator with capacitive drive and sense mechanisms, both electrodes apply electrostatic forces to the resonating structure. The electrostatic force applied by each electrode is as below:

$$
\begin{gathered}
f=\frac{\partial E}{\partial y}=\frac{1}{2}(V-v)^{2} \frac{\partial C}{\partial y} \\
C(y)=\frac{\varepsilon_{o} A}{(g-y)}=\frac{C_{o}}{1-\frac{y}{g}} \Rightarrow \frac{\partial C(y)}{\partial y}=\frac{C_{o}}{g\left(1-\frac{y}{g}\right)^{2}}
\end{gathered}
$$

where $C$ is the capacitance between the electrode and the resonator and $C_{o}$ is its rest value after application of the DC bias voltage, $y$ is the displacement of the resonator towards the electrode, $V$ is the DC bias value of the voltage difference between the resonator and the electrode (polarization voltage), $v$ is the small signal voltage of the electrode, $E$ is the energy stored in the capacitance between the electrode and the resonator, $f$ is the electrostatic force in between the two, $\varepsilon_{0}$ is the permitivity of the surrounding environment and $A$ and $g$ are the capacitance area and the capacitive gap size between the electrode and the resonator respectively. 
Assuming that $v$ and $y$ are small enough so that the device is operating in its linear region, simplifications using Taylor's series, results in the general force equation as below:

$$
f=\frac{1}{2} \frac{C}{g}\left(V^{2}-2 V v+v^{2}+V^{2} \frac{y}{g}-2 V v \frac{y}{g}+v^{2} \frac{y}{g}\right)
$$

For small signal analysis, only the force terms at the drive frequency should be taken into account, therefore:

$$
f=\frac{1}{2} \frac{C}{g}\left(-2 V v+V^{2} \frac{y}{g}\right)
$$

As shown in Eq. 3.4, the force applied by each of the electrodes to the resonator has two components, one caused by the fluctuations of the voltage between the electrode and the resonating structure and the other caused by the fluctuations of the gap size between the electrode and the resonator.

To derive the admittance parameters $y_{11}$ and $y_{21}$, it should be assumed that the sense electrode is shorted to ground so that $v_{s}=0$, therefore the force applied by the sense electrode includes only the displacement induced component:

$$
f_{s}=-\frac{1}{2} \frac{C_{s o}}{g_{s}^{2}} V_{s}^{2} y
$$

The minus sign in Eq. 3.5 is due to the fact that the force applied by the sense electrode is in the opposite direction of the force applied by the drive electrode.

For a beam resonator as a mechanical system with stiffness, effective mass, and damping factor $K, M$ and $D$, and assuming that vibration modes are isolated from each other we have: 


$$
f=M \ddot{y}+D \dot{y}+K y=f_{d}+f_{s}=\frac{1}{2} \frac{C_{d . e f f}}{g_{d}}\left(-2 V_{d} v_{d}+V_{d}^{2} \frac{y}{g_{d}}\right)+\frac{1}{2} \frac{C_{s . e f f}}{g_{s}^{2}} V_{s}^{2} y
$$

It should be noted that to have a precise model for the electrostatically driven resonators, the effective electrode area and consequently the effective drive and sense capacitance values should be calculated. The effective capacitance values depend on the average displacement amplitude of the resonator towards the electrodes. In other words if the electrode is split into a number of small elements, those elements that experience a higher resonator vibration amplitude have more effect on the resonator operation and vice versa. This is due to the fact that the effective stiffness of the beam at each point depends on the displacement amplitude at that point. Looking at different points of the resonator, the whole system has the same amount of energy released and restored in each cycle. Since the potential energy depends on the square of the displacement amplitude $\left(E_{p}=\frac{1}{2} K y^{2}\right)$, the effective stiffness at each point should be proportional to the inverse of the square of the normalized displacement amplitude at that point to result in the same potential energy:

$$
K_{\text {mech.eff }}(x)=\frac{K_{\text {mech.mid }}}{Y(x)^{2}}
$$

Where $K_{\text {mech.eff }}(x)$ is the effective mechanical stiffness at position $x, K_{\text {mech.mid }}$ is the mechanical stiffness of the beam in its central position, and $Y(x)$ is the normalized mode shape function for the first flexural mode of a clamped-clamped beam (Fig. 3.3) resonator having a value of unity at the central point of the beam. 


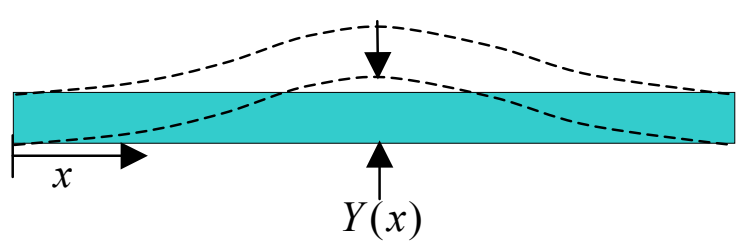

Figure 3.3. Mode shape for the first flexural resonance mode of a clamped-clamped beam resonator. $\mathrm{Y}(\mathrm{x})$ is the normalized displacement function along the beam length having a value of unity in the middle.

Therefore to calculate the overall effect of the electrode, the partial effect of all its elements should be integrated. As expressed in Eq. 3.4, the force applied by each electrode has a voltage dependant component as well as a displacement dependant component. The effect of the displacement amplitude on the effective electrode length for the two force components is different. For both components the stiffness experienced at each point is proportional to the inverse of the square of the displacement amplitude. However for the voltage dependant component, the displacement at each position will be scaled up by the inverse of the normalized displacement at that position when transferred to the center of the beam, therefore the effective force applied by each electrode element will be proportional to $Y(x)$. For clamped-clamped beam resonators operating in their first flexural resonance mode, which is the main focus of this work, assuming that the electrode is placed in the middle of the beam and has a physical length $L_{e}$, the effective length of the electrode for the voltage dependant force term $\left(L_{\text {e.eff }}\right)$ can be calculated as below:

$$
L_{\text {e.eff } 1}=\int_{\frac{L-L_{e}}{2}}^{\frac{L+L_{e}}{2}} Y(x) d x
$$

where $L$ is the length of the beam. 
The displacement dependant component as demonstrated in Eq. 3.6, shows up as a negative stiffness, known as the electrical stiffness and slightly changes the resonance frequency of the resonator. According to Eq. 3.7, since the stiffness at each point is proportional to the inverse square of the normalized displacement, the contribution of the electrode elements at each point will also have the same scaling factor. Therefore the effective length for the displacement induced force component $\left(L_{e . e f f 2}\right)$ can be calculated using the integral in Eq. 3.9:

$$
L_{\text {e.eff } 2}=\int_{\frac{L-L_{e}}{2}}^{\frac{L+L_{e}}{2}} Y(x)^{2} d x
$$

From now on, the electrode effective lengths will be used to calculate the effective electrode area $\left(A_{\text {d.eff } 1,2}\right.$ and $\left.A_{\text {s.eff } 1,2}\right)$ and effective drive and sense capacitances $\left(C_{\text {d.eff } 1,2}\right.$ and $\left.C_{\text {s.effl, }, 2}\right)$.

Solving Eq. 3.6 using Laplace transform and replacing the drive and sense capacitances with their effective values results in:

$$
\frac{y(s)}{v_{d}(s)}=-\frac{\left(\frac{C_{d . e f f 1} V_{d}}{K g_{d}}\right)}{\left(\frac{s^{2}}{\omega_{m}{ }^{2}}+\frac{s}{\omega_{m} Q}+\left(1-\frac{V_{d}{ }^{2} C_{d . e f f 2}}{K g_{d}{ }^{2}}-\frac{V_{s}^{2} C_{s . e f f 2}}{K g_{s}{ }^{2}}\right)\right)}
$$

where $\omega_{m}=\left(\frac{K}{M}\right)^{\frac{1}{2}}$, and $Q=\left(\frac{\sqrt{K \cdot M}}{D}\right)$. It should be noted that since the effective electrode lengths are calculated with respect to the central point of the beam, the stiffness $(K)$, and mass $(M)$ used in Equations 3.6, and 3.10, are the stiffness and mass of the beam in the middle. 
Equation 3.10 expresses the vibration amplitude of the resonator in terms of the applied drive and polarization voltages as well as the mechanical and geometric properties of the resonator. Vibration of the resonator results in fluctuations of the capacitive gap size and consequently the capacitor values in between the resonating body and the electrodes. The change in the capacitances in turn results in fluctuation of the electric charge stored in the sense and drive capacitors causing an alternative current in both sense and drive electrodes as shown in Eq. 3.11.

$$
I_{i}=\frac{\partial q}{\partial t}=\frac{\partial\left(C_{d}\left(v_{d}-V_{d}\right)\right)}{\partial t}=\frac{\partial C_{d, e f f 1}}{\partial t}\left(v_{d}-V_{d}\right)+C_{d} \frac{\partial v_{d}}{\partial t} \approx-\frac{C_{d, e f f 1}}{g_{d}} \cdot \frac{\partial y}{\partial t}\left(v_{d}-V_{d}\right)+C_{d} \frac{\partial v_{d}}{\partial t}
$$

Assuming that $v_{d}$ is much smaller than $V_{d}$ and applying Laplace transform to Eq. 3.11 results in:

$$
I_{i}(s)=\frac{s y(s) C_{d, e f f 1}}{g_{d}} V_{d}+C_{d} s v_{d}(s)
$$

It should be noted that since the change in the capacitance and consequently the current induced at each point of the electrode is proportional to the displacement amplitude at that point, $C_{\text {effi }}$ is to be used for calculation of displacement induced current term. Substituting $x(s)$ from Eq. 3.10 into Eq. 3.12 results in:

$$
y_{i}=y_{11}=\left.\frac{I_{i}(s)}{v_{i}(s)}\right|_{v_{o}=0}=\frac{C_{d, \text { eff } 1} V_{d}\left(\frac{C_{d, e f f 1} V_{d}}{K g_{d}}\right)}{\left(\frac{s}{\omega_{m}{ }^{2}}+\frac{1}{\omega_{m} Q}+\left(1-\frac{V_{d}{ }^{2} C_{d, e f f 2}}{K g_{d}{ }^{2}}-\frac{V_{s}{ }^{2} C_{s, e f f 2}}{K g_{s}{ }^{2}}\right) \frac{1}{s}\right) g_{d}}+C_{d} s
$$

As shown in Eq. 3.13, the equivalent electrical admittance at the input node of the resonator includes a capacitor equal to the drive capacitor in parallel with a series RLC with the following values: 


$$
\begin{aligned}
& L_{i}=\frac{K g_{d}{ }^{2}}{C_{d, e f f 1}{ }^{2} V_{d}^{2} \omega_{m}{ }^{2}} \\
& C_{i}=\frac{C_{d, e f f 1}{ }^{2} V_{d}{ }^{2}}{K g_{d}{ }^{2}\left(1-\frac{V_{d}{ }^{2} C_{d, e f f 2}}{K g_{d}{ }^{2}}-\frac{V_{s}{ }^{2} C_{s, e f f 2}}{K g_{s}{ }^{2}}\right)} \\
& R_{i}=\frac{\sqrt{K M} g_{d}{ }^{2}}{Q C_{d, \text { eff } 1}{ }^{2} V_{d}{ }^{2}}=\frac{K g_{d}{ }^{2}}{Q \omega_{m} C_{d, \text { eff } 1}{ }^{2} V_{d}{ }^{2}}=\frac{D g_{d}{ }^{2}}{C_{d, \text { eff } 1}{ }^{2} V_{d}{ }^{2}}
\end{aligned}
$$

The same derivations can be performed for the other port of the resonator (sense electrode) resulting in similar equation for $y_{22}$ with all drive electrode parameters $\left(V_{d}, g_{d}\right.$, and $\left.C_{d . e f f 1,2}\right)$ replaced by sense electrode parameters $\left(V_{s}, g_{s}\right.$, and $\left.C_{s . e f f 1,2}\right)$.

In order to derive the trans-conductance parameters $\left(y_{12}\right.$, and $\left.y_{21}\right)$, the current generated in one electrode while an electrical signal is applied to the other electrode should be calculated. For $y_{21}$ it can be done by replacing $g_{d}, C_{d . e f f}$, and $V_{d}$ with $g_{s}, C_{s . e f f}$, and $V_{s}$ in Eq. 3.12. Since the sense electrode is assumed to be grounded, the second term of the equation will be zero:

$$
I_{o}(s)=\frac{s y(s) C_{s . e f f 1}}{g_{s}} V_{s}
$$

substituting $y(s)$ from Eq. 3.10:

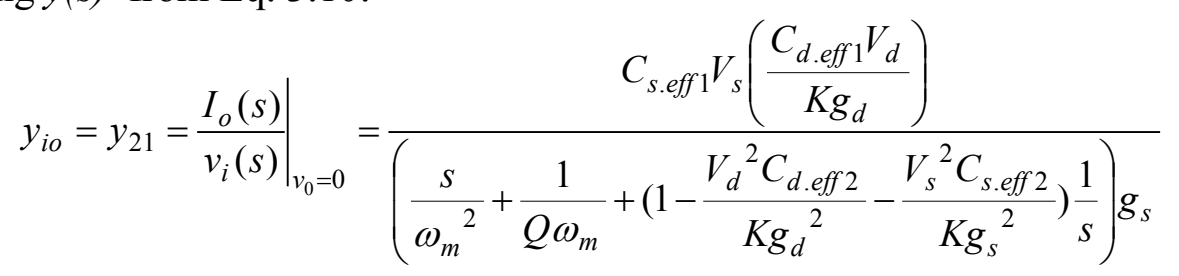

It is concluded from Eq. 3.18 that the current generated in the output port by the input voltage is equal to the current produced by the same voltage in a series RLC tank with the following values: 


$$
\begin{aligned}
& L_{i o}=\frac{K g_{d} g_{s}}{C_{d . e f f ~} C_{s . e f f 1} V_{d} V_{s} \omega_{m}{ }^{2}}=\frac{L_{i}}{T_{i o}} \\
& C_{i o}=\frac{C_{d . e f f 1} C_{s . e f f 1} V_{d} V_{s}}{K g_{d} g_{s}\left(1-\frac{V_{d}{ }^{2} C_{d . e f f 2}}{K g_{d}{ }^{2}}-\frac{V_{s}^{2} C_{s . e f f 2}}{K g_{s}{ }^{2}}\right)}=C_{i} \cdot T_{i o}
\end{aligned}
$$

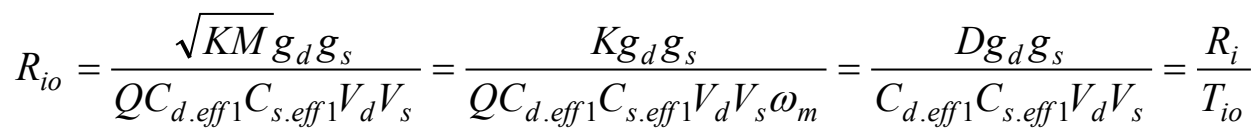

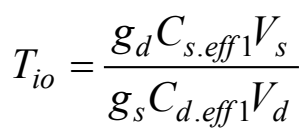

Same equations can be derived for the trans-conductance from output to input resulting in:

$$
y_{o i}=y_{12}=y_{i o}=y_{21}=T_{i o} y_{i}=T_{o i} y_{o}
$$

where $T_{i o}=T_{o i}{ }^{-1}$.

Based on the derived y-parameters, the equivalent circuit model for a two port clamped-clamped beam resonator will be as shown in Fig. 3.4.

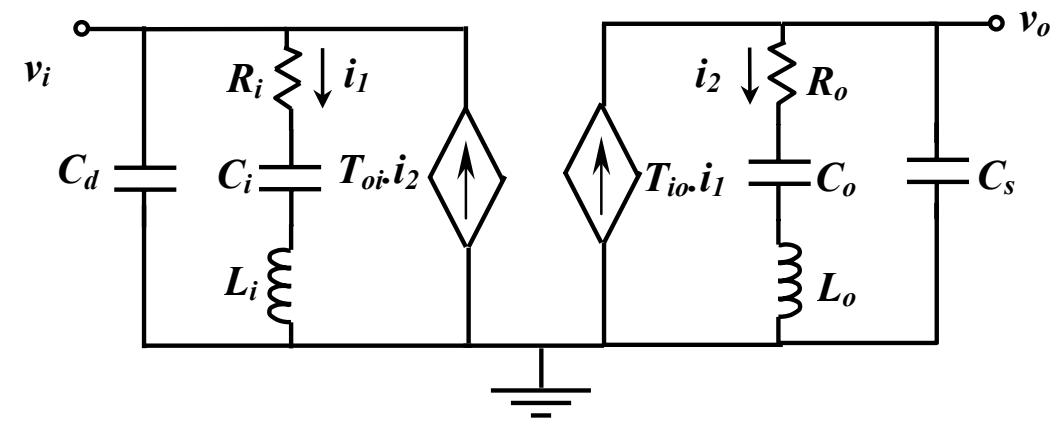

Figure 3.4. Equivalent small signal model for a two-port micro-mechanical beam resonator. 
The equivalent circuit model can be expressed in an alternative way. Since the two trans-admittance parameters $\left(y_{12}\right.$ and $\left.y_{21}\right)$ are equal and in the form of the admittance of a series RLC tank, the equivalent circuit model includes a series RLC connecting the two ports. On the other hand, the input and output admittances $\left(y_{11}\right.$ and $\left.y_{22}\right)$ have the same transfer functions as the trans-admittance parameters scaled by the constant factors, $T_{i o}$ and $T_{o i}$. Adding transformers with transformation ratios $\sqrt{T_{i o}}:: 1$ to the input and output ports of the RLC tank, will scale the input and output impedances to the required values without changing the trans-admittance parameters. Therefore the equivalent circuit model shown in Fig 3.5 also has all the admittance parameters derived for the resonator and can be used for describing the dynamic behavior of the beam resonators.

It is worth mentioning that when interconnect pads are added to the input and output of the resonator, the capacitances related to the pads should be included in $C_{d}$ and $C_{s}$.

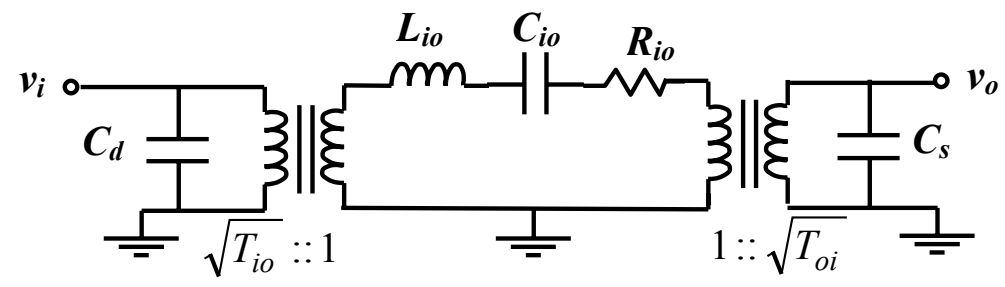

Figure 3.5. Alternative demonstration of the equivalent small signal model for a two port micro-mechanical beam resonator.

In case of a completely symmetric resonator (similar drive and sense electrodes), the terminating transformers will have a ratio of $1:: 1$ and can be eliminated, so that the equivalent circuit will be a single series RLC tank with two terminating capacitors. 


\subsubsection{Electrostatic Tuning of Micromechanical Beam Resonators}

One of the useful features of capacitive resonators with parallel plate actuators is the frequency tuning capability of such resonators. As it was discussed earlier, the displacement dependant force component applied by the each electrode acts as a negative electrical stiffness added to the resonator. The value of the negative stiffness depends on the polarization voltage and therefore changing the polarization voltage of the resonator, changes the total stiffness and consequently the operating frequency of the resonator. The physical explanation of this phenomenon is as following: as the distance of the resonator to one electrode decreases, the electrostatic force attracting the resonator to the electrode increases, at the same time the force applied by the other electrode decreases and vice versa. Therefore this force component is in phase with the displacement of the resonator (helps the movement of the resonator). On the other hand the mechanical force of the resonator is in the opposite phase with the displacement and proportional to it, so the displacement dependant electrostatic force seems to be subtracted from the mechanical force applied by the mechanical stiffness, and can be modeled by a negative effective stiffness.

Based on the derived values of equivalent $L$ and $C$ for the resonators, the tuned frequency including the effect of the electrical stiffness will be as given by Eq. 3.24:

$$
f_{s}=\frac{1}{2 \pi \sqrt{L C}}=f_{m} \sqrt{\left(1-\frac{V_{p}{ }^{2} C_{d . e f f 2}}{K g_{d}{ }^{2}}-\frac{V_{p}{ }^{2} C_{s . e f f 2}}{K g_{s}{ }^{2}}\right)}
$$




\section{CHAPTER IV}

\section{ELECTRICAL COUPLING OF MEM RESONATORS FOR BANDPASS FILTER SYNTHESIS}

\subsection{Active Coupling of Resonators}

The first approach used for implementation of electrically coupled high order MEM filters in this work is electrical cascading of resonators using active electronic components in between the resonators (Fig. 4.1) [32]. The electrical cascading of resonators with buffers or amplifiers in between (to eliminate the loading effect of resonators on each other) results in multiplication of the transfer functions and an overall higher order transfer function with several pairs of conjugate poles.

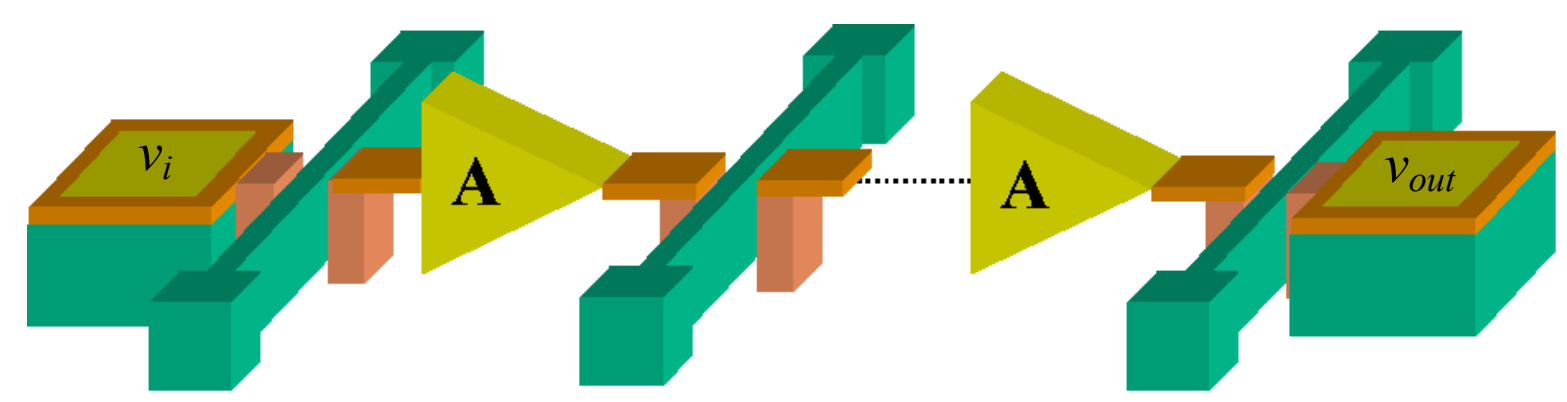

Figure 4.1. Schematic diagram of an electrically cascaded resonant system comprised of micromechanical resonators interfaced with active electrical components in between.

When all the stages have equal center frequencies, cascading of the resonators will result in order multiplication of poles, which can be interpreted as a higher overall quality factor. Mathematically, it can be shown that if $n$ identical $2^{\text {nd }}$ order resonators 
with individual quality factors of $Q_{i}$ are cascaded, the resultant $\mathrm{Q}$ factor of the cascade is equal to:

$$
Q_{\text {total }}=\frac{Q_{i}}{\sqrt{10^{0.3 / n}-1}} \rightarrow Q_{\text {total }} \cong 1.2 \sqrt{n} Q_{i} \text { if } n>>1
$$

This concept can be used to increase the equivalent quality factor of MEM resonators for filtering or frequency synthesis applications, in case their intrinsic Q is not high enough. In addition, smaller shape factors (higher selectivity) can be obtained from the cascaded resonators, where shape factor is the ratio of the bandwidth in which the insertion loss is smaller than a specific value to the $3 \mathrm{~dB}$ bandwidth of the filter (Fig. 4.2).

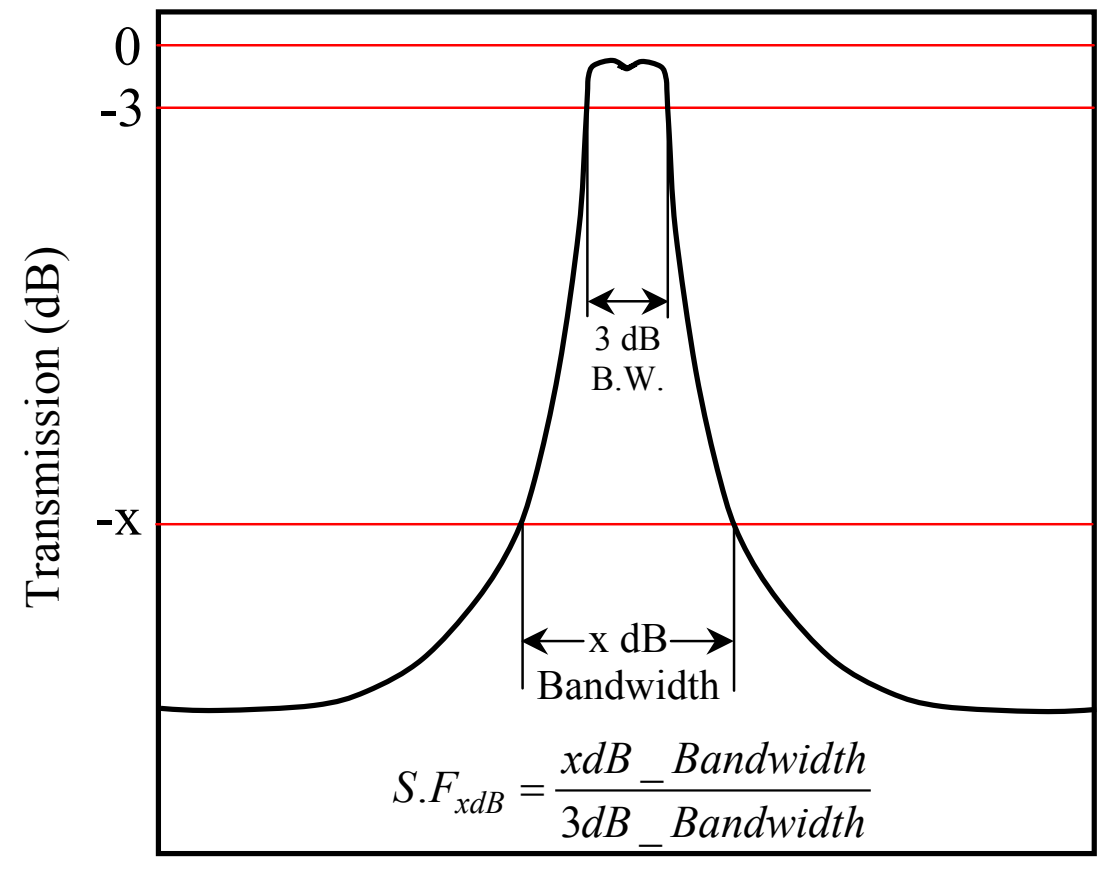

Frequency

Figure 4.2. Frequency response of a typical bandpass filter and definition of the shape factor.

Shape factor of electrically cascaded resonators with equal center frequencies can be calculated using Eq. 4.2. According to Eq. 4.2, shape factor for the cascaded 
resonators is determined only by the order of the system, independently of the quality factor of individual resonators.

$$
S . F_{40 d B}=\sqrt{\frac{10^{4 / n}-1}{10^{0.3 / n}-1}} \rightarrow 1 \text { as n becomes large }
$$

Figure 4.3a illustrates simulation results for cascaded resonators with different orders showing the overall Q amplification by increasing the order of the system. The comparison between cascaded resonators with different number of stages but identical overall Q (Fig. 4.3b) confirms that despite having equal quality factors, higher order cascades provide sharper roll-off and higher selectivity (lower shape factor).

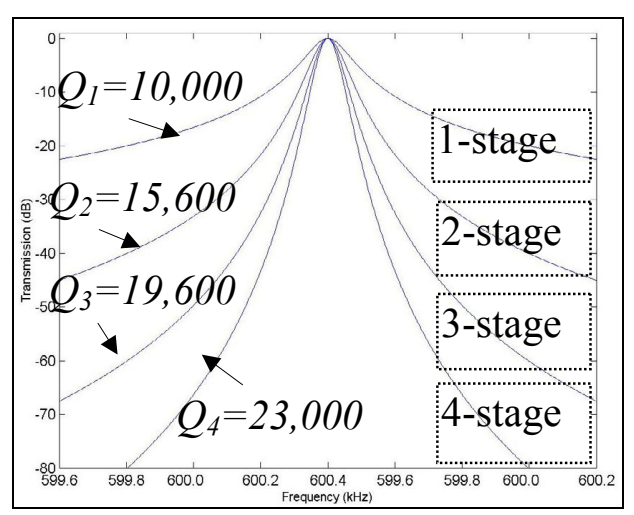

(a)

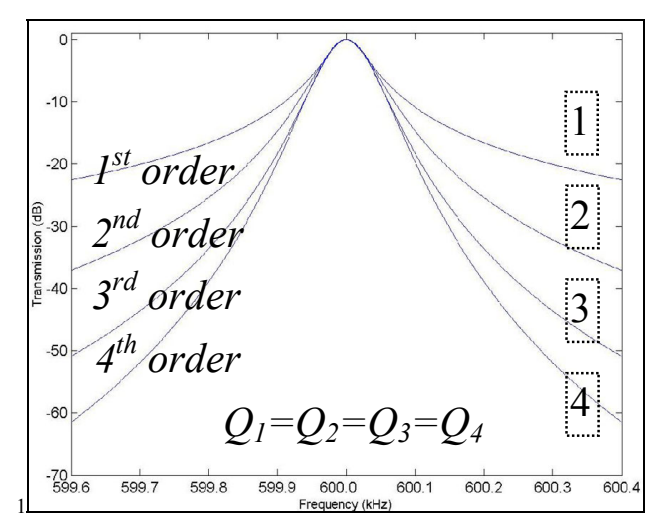

(b)

Figure 4.3. (a) Simulation results for cascaded resonators with individual $Q=10,000$ $(600 \mathrm{kHz})$. (b) Simulation results of cascaded resonators with identical overall quality factors $\left(Q_{\text {overall }}=10,000\right) \&$ different orders.

To achieve larger bandwidths without sacrificing the sidewall sharpness in electrically cascaded micromechanical filters, one can take advantage of the frequency tunability of capacitive resonators. Introducing a slight mismatch between the center frequencies of cascaded resonators results in separation of resonance peaks and hence a 
wider bandwidth. However, center frequencies of cascaded devices should be close enough to avoid excessive attenuation of each stage by the other stages.

\subsection{Capacitive Coupling of Resonators}

The electrical cascading approach described in the previous section can be deployed for realization of higher order micromechanical filters. However, for filter bandwidths much larger than the bandwidth of individual resonators, large signal loss will be introduced by the stages with different resonance frequencies. In addition, integration of the micromechanical resonators with interfacing active electronic components for implementation of fully integrated active coupled filters in not a trivial task.

The capacitive coupling technique for implementation of higher order microelectromechanical filter arrays uses capacitors as the coupling elements, providing the ability to implement fully integrated filters without the need for integration with active electronics [32]. Capacitive coupling is the electrical counterpart of the mechanical coupling approach described in the previous chapter.

As depicted in Fig. 4.4, a capacitively coupled filter array consists of micromechanical resonators cascaded with shunt capacitors to ground in between every two adjacent resonators. In such systems, the interaction of the coupling capacitors and the equivalent RLC tanks of the resonators results in several resonance modes and consequently a multiple-order bandpass frequency response for the system. 


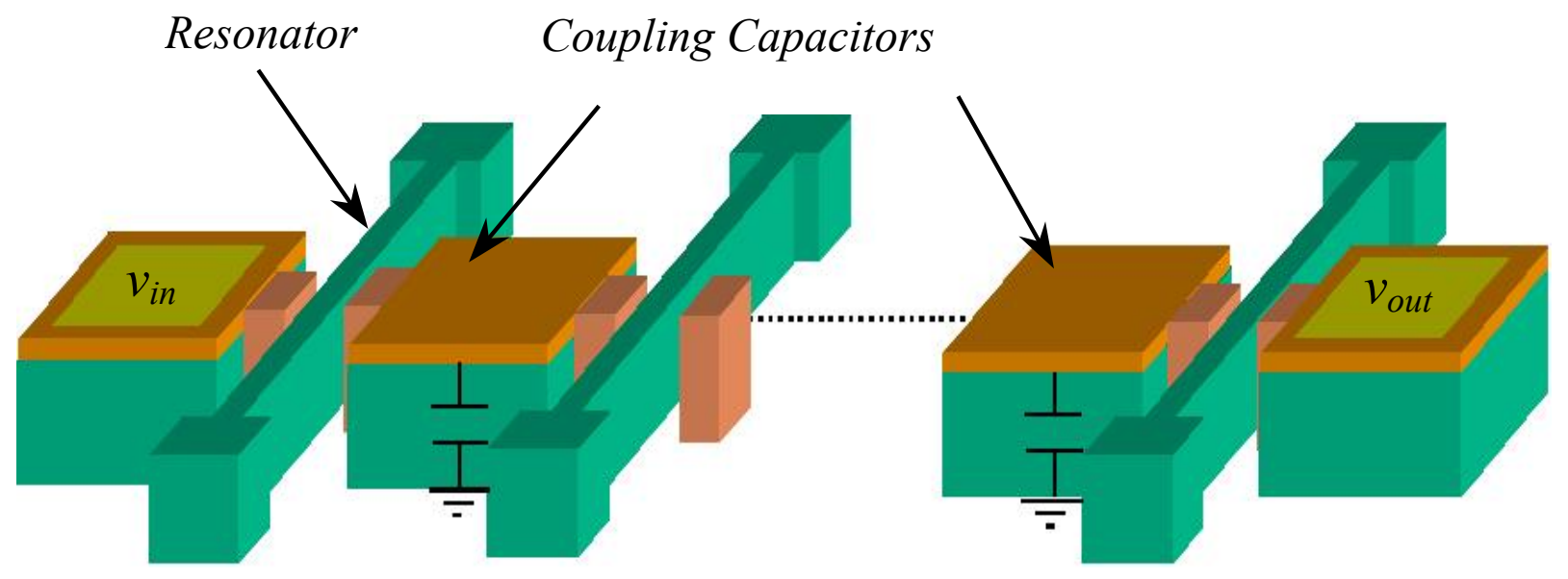

Figure 4.4. Schematic diagram of a capacitively-coupled microelectromechanical bandpass filter.

To describe the operation of a capacitively coupled filter lets assume a second order filter consisting of two similar resonators coupled with one coupling capacitor in between the two. Capacitive coupling of the two resonators with identical center frequencies $\left(f_{0}\right)$, quality factors $(Q>1000)$, and motional resistances $(R)$, will introduce a new resonance mode to the system in addition to the inherent resonance mode of the individual resonators at $f_{0}$. The new resonance mode is a result of resonance of each resonator with half of the coupling capacitor added to them in series. The resulting resonance frequency will therefore be:

$$
f_{1}=f_{0} \sqrt{\frac{1+\pi f_{0} C_{c} R Q}{\pi f_{0} C_{c} R Q}}
$$

where $C_{c}$ is the value of the coupling capacitor. The electrical equivalent model of the filter is shown in Fig. 4.5. For simplicity, each resonator is assumed to be symmetric and therefore represented by a series RLC tank. Looking at the frequency response of the 
filter, the first resonance occurs at the mechanical resonant frequency of the individual resonators. At the $1^{\text {st }}$ resonance mode, as shown in Fig. 4.5, the two resonators resonate in phase and the coupling capacitor has no contribution to the response (while $\mathrm{C}_{\mathrm{c}}$ is being charged by the first resonator, the other resonator is discharging it). At the $2^{\text {nd }}$ resonance mode $\left(f_{l}\right)$, the two resonators resonate with a $180^{\circ}$ phase difference and hence the coupling capacitor comes into the game (it is being charged and discharged at the same time by both resonators). Due to the symmetry of the equivalent circuit, it can be reduced to a half circuit with one resonator and a series capacitor $C_{d} / 2$ to ground. The series capacitor $\left(C_{c} / 2\right)$ added to the resonator, reduces the total capacitance of the resulting RLC tank, causing the second resonance mode to have a higher resonance frequency given by Eq. 4.3.
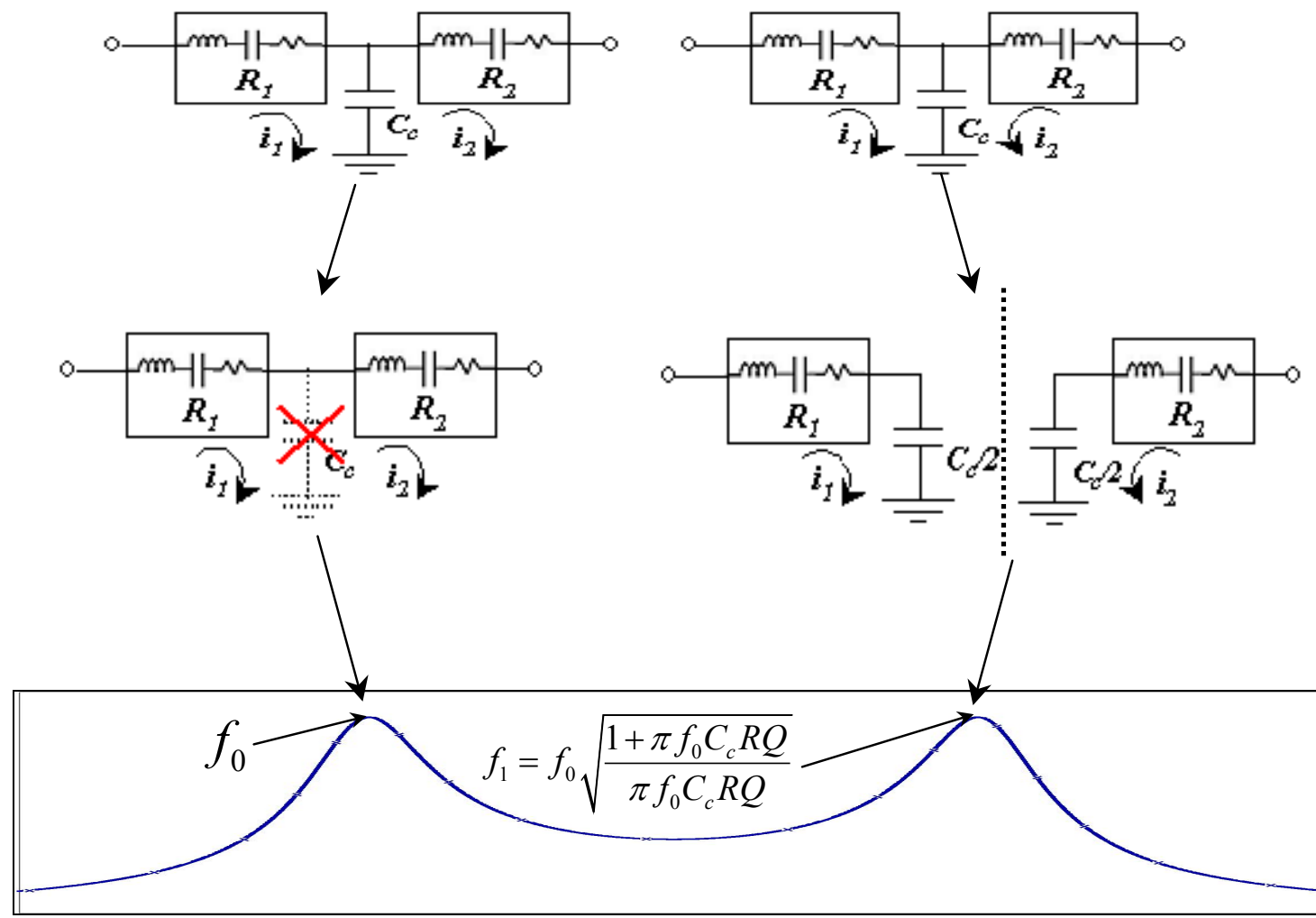

Figure 4.5. Electrical schematic diagram of a second order capacitively coupled resonant system and its frequency response. 
For a three-resonator system with two coupling capacitors, as shown in Fig. 4.6 the case is more complicated. Similar to the second order example, in the first mode of the third order filter, all the resonators resonate in phase and the coupling capacitors have a negligible contribution. At the second resonance mode, the two terminating resonators resonate out of phase while the resonator in the middle remains stationary, having no contribution to the response. In this case, each resonator will have one coupling capacitor in series resulting in a higher resonance frequency of:

$$
f_{1}=f_{0} \sqrt{\frac{1+2 \pi f_{0} C_{c} R Q}{2 \pi f_{0} C_{c} R Q}}
$$

In the third resonance mode, each resonator resonates $180^{\circ}$ out of phase with respect to its adjacent resonator(s). Therefore the coupling capacitors are shared between the three of them. To have equal resonance frequencies for all the resonators with the added capacitors, two third of each coupling capacitor is to be added to the resonator in the middle while the remaining one third of each capacitor is added to its adjacent terminating resonator. Therefore, the resulting resonance frequency for this mode is:

$$
f_{2}=f_{0} \sqrt{\frac{3+2 \pi f_{0} C_{c} R Q}{2 \pi f_{0} C_{c} R Q}}
$$




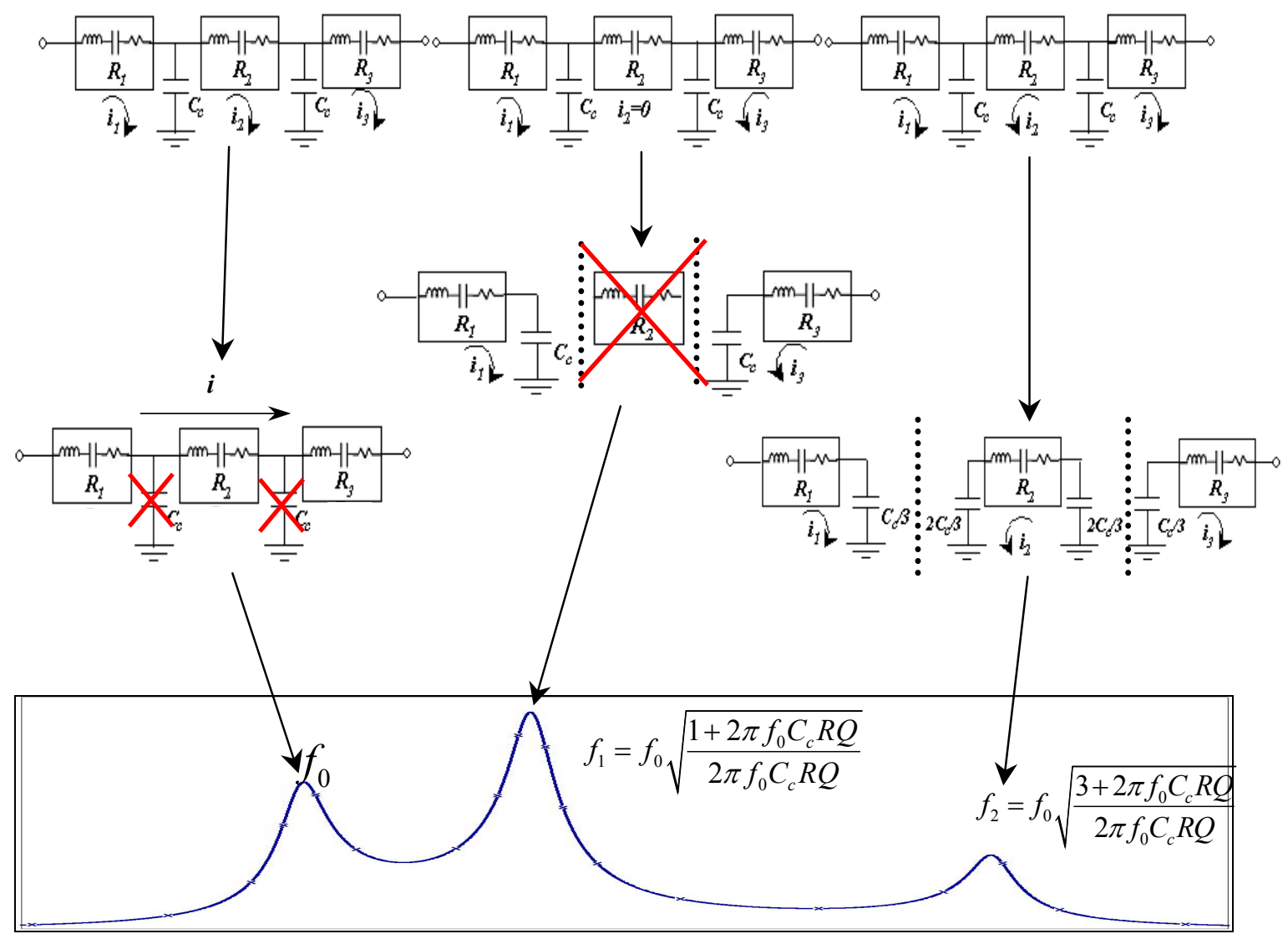

Figure 4.6. Electrical schematic diagram of a third order capacitively coupled resonant system and its frequency response.

The asymmetry in the frequency response of the $3^{\text {rd }}$ order filter is due to the fact that the two terminating resonators have only one coupling capacitor adjacent to them while the one in the middle is terminated with two coupling capacitors. This asymmetry can be compensated by slight frequency tuning of the terminating resonators with respect to the rest of the resonators. However, tuning of the frequency of the resonators to different values, results in an increased insertion loss. A better solution to this problem is 
to use a closed chain of coupled resonators [17] as shown in Fig. 4.7 to have complete symmetry for all the resonators.

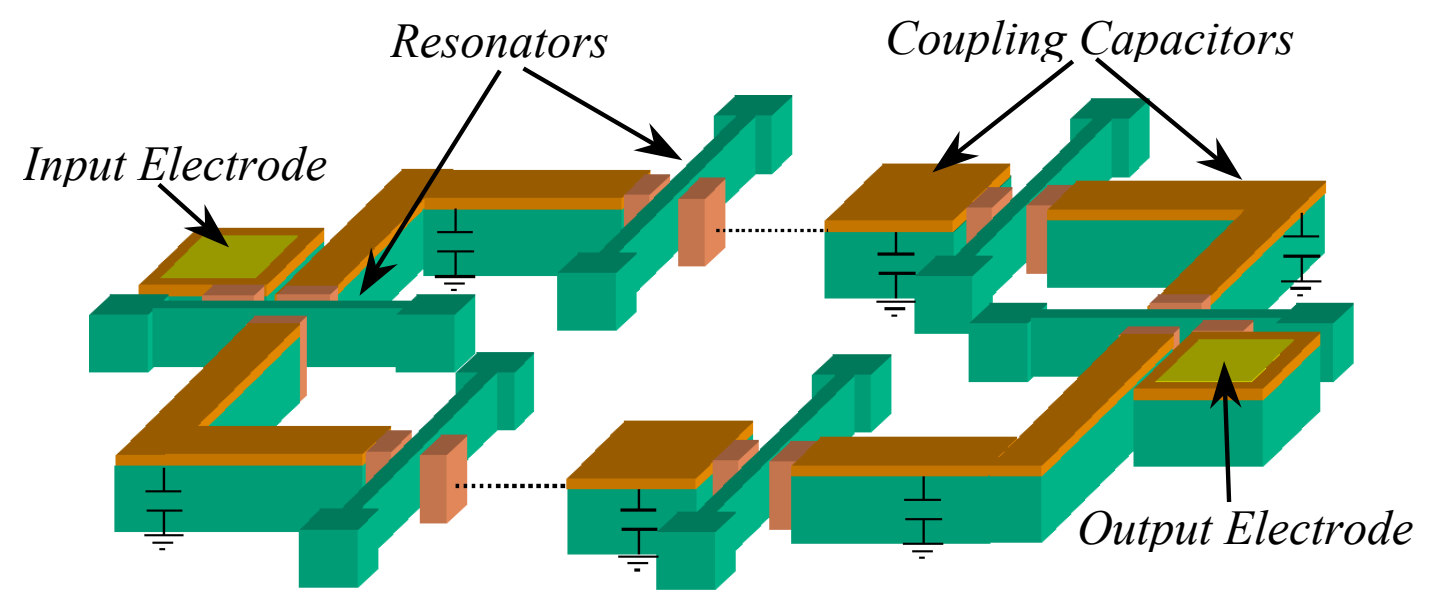

Figure 4.7. Schematic diagram of a closed loop array of capacitively-coupled microelectromechanical resonators.

Similar to the mechanically coupled filters, the passband of the capacitively coupled filters is flattened by adding terminating resistors (Fig. 4.8). The terminating resistors reduce the quality factor of the resonators by introducing additional damping. In a real case, the terminating resistors are the output and input resistance of the system components adjacent to the filter.
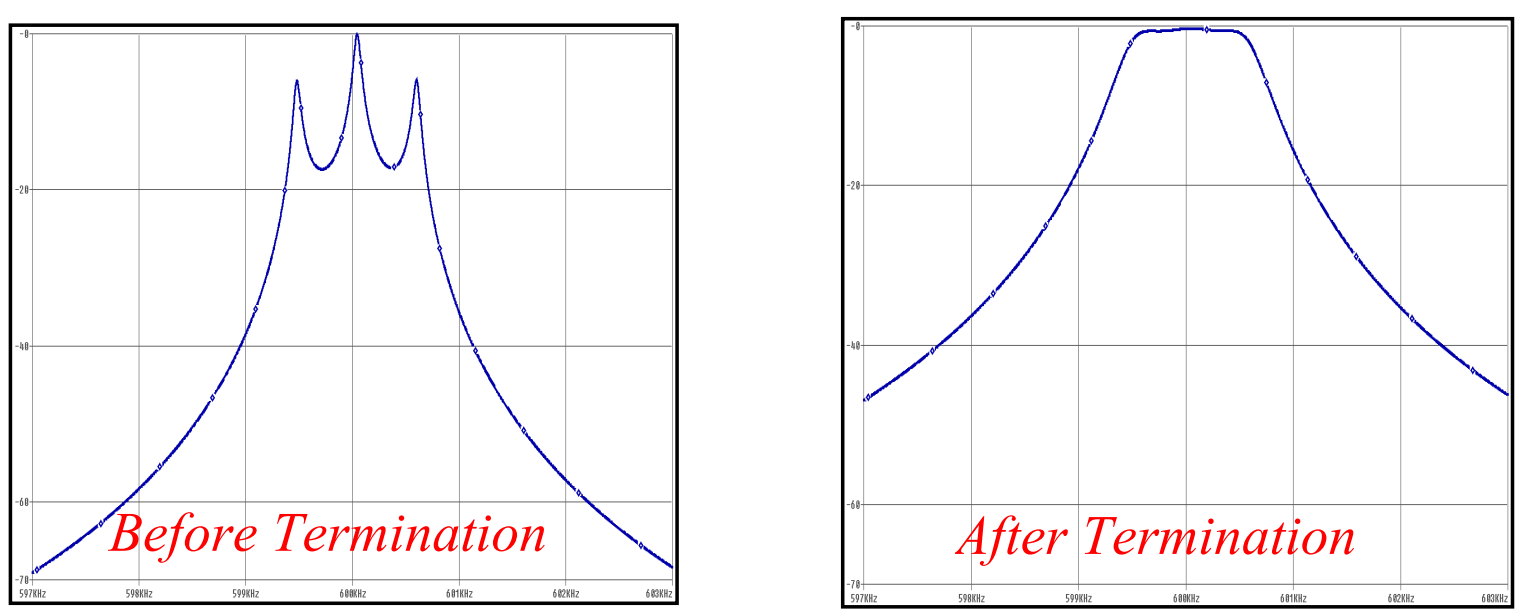

Figure 4.8. Frequency response of a typical third order capacitively coupled filter before and after adding terminating resistors. 


\subsubsection{Insertion Loss in Capacitively Coupled Filters}

The insertion loss of a capacitively coupled filter (assuming ideal lossless coupling capacitors) is determined by the order of the filter, the equivalent motional resistance of the resonators and the terminating resistor values that flatten the filter passband. In a real case when the filter is incorporated in an electrical system, the terminating resistors are input and output equivalent resistance of the electronic stages adjacent to the filter (i.e. low noise amplifiers). With good approximation an $\mathrm{n}^{\text {th }}$ order filter at resonance is equivalent to $\mathrm{n}$ resistors in series each having value of $R_{r}$ (equivalent motional resistance of the resonator). Therefore:

$$
\text { Insertion }-\operatorname{Loss}(d B)=20 \log \left(\frac{n R_{r}+2 R_{\text {term }}}{2 R_{\text {term }}}\right)
$$

where $R_{\text {term }}$ is the terminating resistor value. The required terminating resistance value depends on filter bandwidth, maximum tolerable passband ripple, and motional resistance of the resonators. For resonators with similar design parameters (gap size, electrode area, and polarization voltage) resulting in equal equivalent motional capacitance $\left(C_{m}\right)$ and consequently equal filter bandwidths, the motional resistance is determined by the quality factor of the resonators. Therefore filter insertion loss is dependant on the quality factor of individual resonators. Figure 4.9 illustrates simulation results of second and third order capacitively coupled electromechanical filters at $600 \mathrm{kHz}$ with different resonator quality factors, showing dependence of filter insertion loss on the $\mathrm{Q}$ of individual resonators.

For the specific filter characteristics of Fig. 5, coupling capacitors of $0.15 \mathrm{pF}$ and $0.2 \mathrm{pF}$ are used that can be easily fabricated on-chip in any MEMS or CMOS process. 
According to Equations 4.3, 4.4, and 4.5, the value of the coupling capacitors can be extracted from the resonators motional resistance, operating frequency, required filter bandwidth, and tolerable passband ripple.
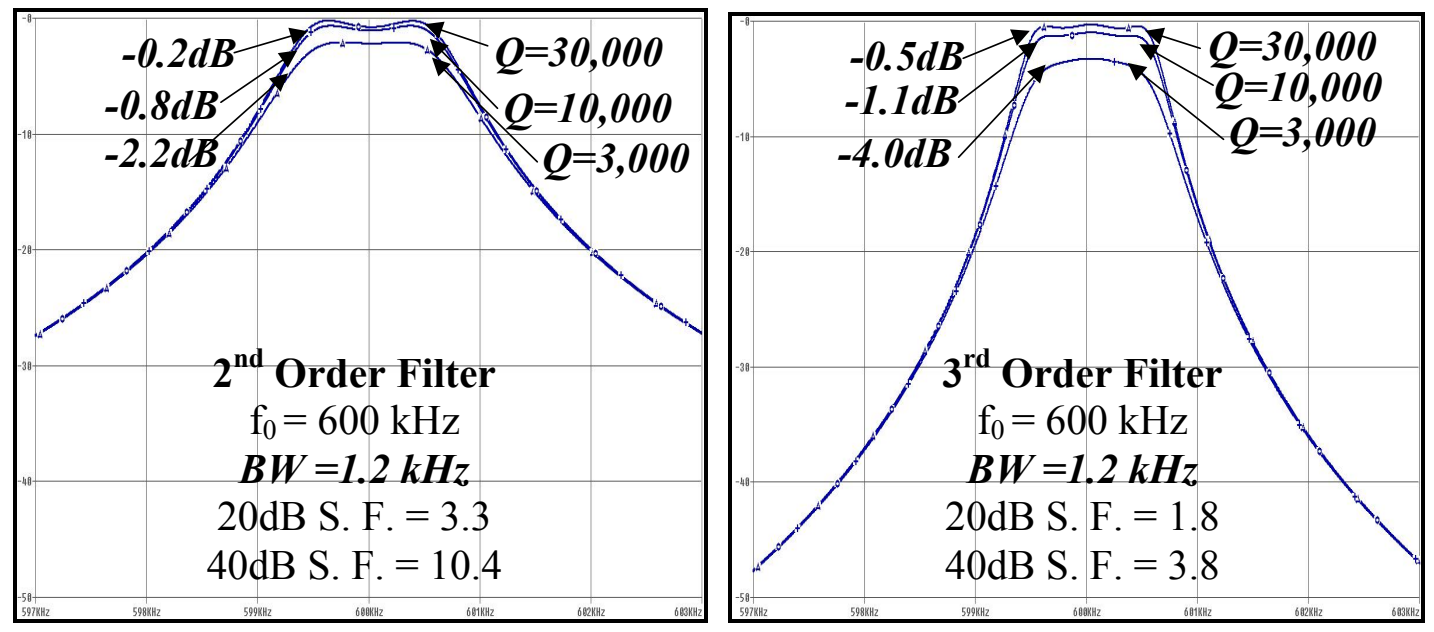

Figure 4.9. Simulation results for $600 \mathrm{kHz}$ capacitively-coupled $2^{\text {nd }} \& 3^{\text {rd }}$ order filters. A higher resonator $\mathrm{Q}$ results in a lower insertion loss. Coupling cap: $\mathrm{C}_{\mathrm{c}}=0.15 \mathrm{pF}$ ( $2^{\text {nd }}$ order $)$; $\& \mathrm{C}_{\mathrm{c}}=0.2 \mathrm{pF}\left(3^{\mathrm{rd}}\right.$ order $)$.

\subsubsection{Lossy Coupling Capacitors}

Figure 4.10 shows the simulation results with non-ideal (lossy) coupling capacitors. The finite $\mathrm{Q}$ of the coupling capacitors does not have a significant effect on the first resonance peak but its attenuation effect becomes more pronounced in higher resonance modes. This is due to the fact that the coupling capacitors have a negligible effect on the filter operation in the first resonance mode and become more involved in the higher resonance modes. 


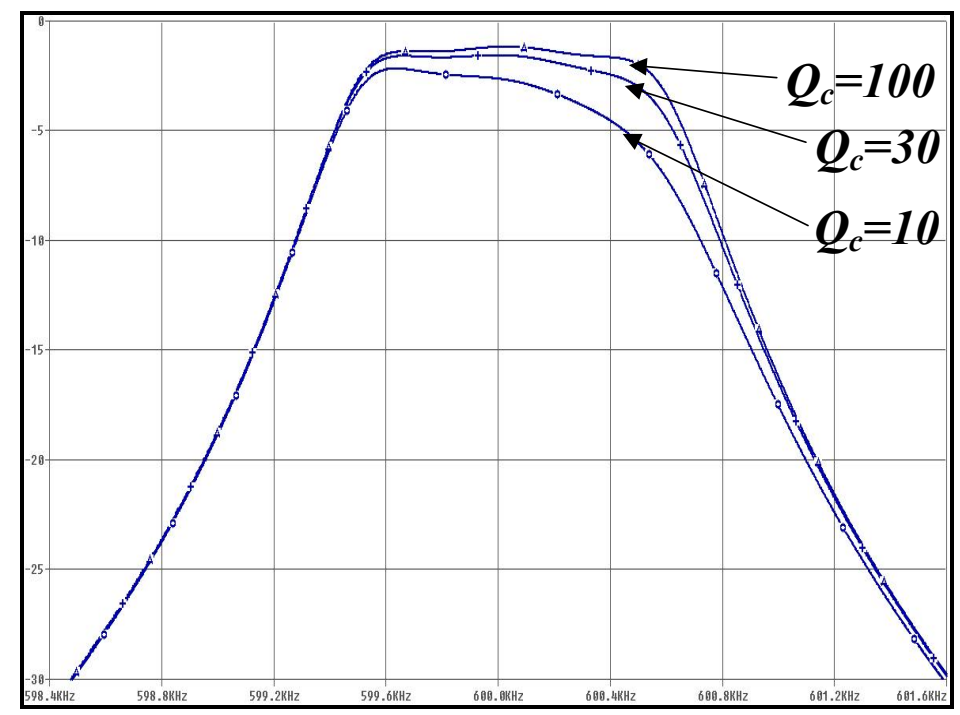

Figure 4.10. Effect of finite $Q$ of the coupling capacitors on the frequency response of the third order capacitively coupled filter of Fig.4.9b with resonator $Q=10,000$.

\subsection{Electrostatic Coupling of Resonators}

The alternative electrical-based passive coupling technique for implementation of high-order bandpass filters from microelectromechanical resonators presented here is electrostatic coupling of the resonators. The electrostatic coupling approach eliminates the need for any physical coupling element in between the resonators and provides the highest degree of tunability and design flexibility among the available filter synthesis approaches.

In this approach, the electrostatic force between the resonating bodies of two closely spaced microresonators causes coupling and results in a higher order resonant system [33]. Compared to the previously discussed capacitive coupling approach [32], the electrostatic coupling provides even more tunability and design flexibility and 
alleviates some of the problems associated with capacitive coupling (such as DC biasing of the coupling capacitor).

Figure 4.11 shows the schematic diagram of an electrostatically coupled beam filter array comprised of a number of resonators separated by small coupling gaps in between.

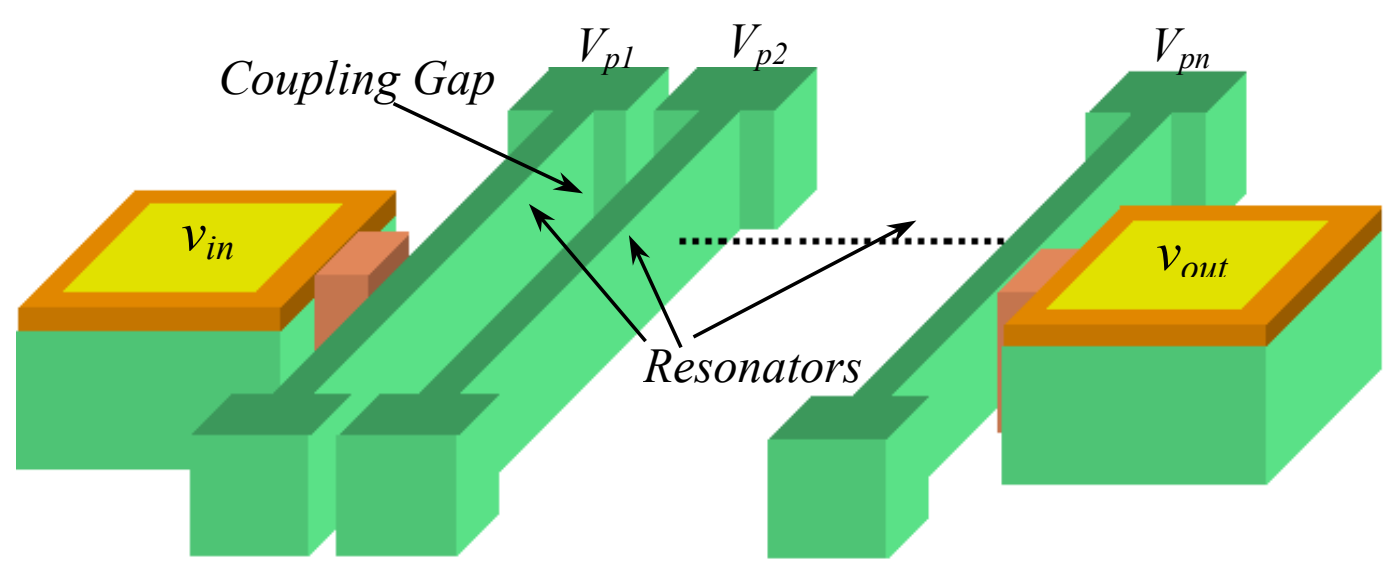

Figure 4.11. Schematic diagram of an electrostatically coupled beam filter array.

\subsubsection{Electrostatic Coupling Force}

The difference in the DC polarization voltages $\left(\mathrm{V}_{\mathrm{p}}\right)$ applied to the adjacent resonators results in an electrostatic force between them. Assume two closely spaced clamped-clamped beam resonators biased at different polarization voltages. For analytical derivation of the coupling strength between the two resonators, each beam is divided into small elements of length $d x$ each, as shown in Fig. 4.12. The electrostatic force in between each pair of corresponding adjacent elements on the two beams is given by: 


$$
d f_{c}=\frac{\partial d E_{c}}{\partial y}=\frac{1}{2}\left(\Delta V_{p}\right)^{2} \frac{\partial d C_{c}}{\partial y}
$$

where $d f_{c}$ is the electrostatic coupling force between the two elements, $d E_{c}$ is the energy stored in the capacitor between the two elements, $y$ is the total displacement of the elements towards each other along the vibration axis of the resonators, $\Delta V_{p}$ is the difference between the two polarization voltages applied to the resonators, and $d C_{c}$ is the value of the capacitor in between the two adjacent elements. Each pair of adjacent elements can be considered as a parallel plate capacitor in which the interelectrode gap spacing changes due to the vibrations of the resonators. Assuming small vibration amplitudes, $\frac{\partial d C_{c}}{\partial y}$ is given by:

$$
\frac{\partial d C_{c}}{\partial y} \approx \frac{d C_{c o}}{g_{c}}\left(1+\frac{y}{g_{c}}\right)
$$

where $g_{c}$ is the coupling gap size in between the resonators, and $d C_{c o}$ is the rest value of $d C_{c}$. Therefore, the electrostatic force can be expressed as:

$$
d f_{c}=\frac{1}{2} \frac{d C_{d o}\left(\Delta V_{p}\right)^{2}}{g_{c}}\left(1+\frac{y}{g_{c}}\right)
$$

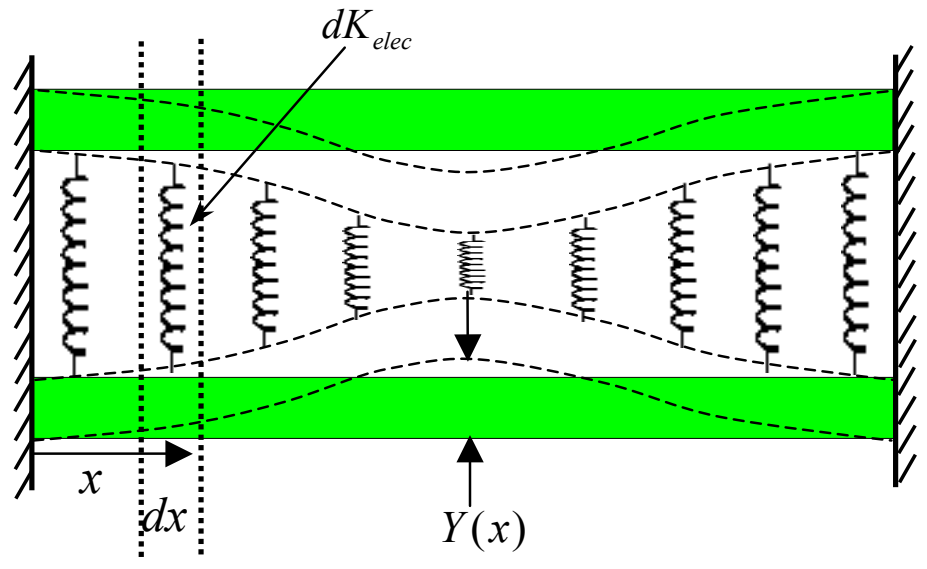

Figure 4.12. Schematic diagram of two electrostatically coupled clamped-clamped beam resonators. 
According to Eq. 4.9, the electrostatic force has a position dependent component (in addition to its constant component) that is in-phase with the displacement of the resonators towards each other. On the other hand, the force applied by the mechanical stiffness of the structure has a $180^{\circ}$ phase shift with the displacement of the resonators. Hence, the position dependent electrostatic force can be modeled as a negative stiffness between the two elements. The electrostatic negative stiffness that exists between every two adjacent elements (represented by springs in Fig. 4.11) is therefore expressed by:

$$
d K_{\text {elec }}=-\frac{\partial d f_{c}}{\partial y}=-\frac{\left(\Delta V_{p}\right)^{2} \varepsilon h_{r} d x}{g_{c}{ }^{3}}
$$

where $h_{r}$ is the height of the resonators and $\varepsilon$ is the permitivity of the surrounding environment. This effect is similar to the electrostatic frequency tuning of parallel plate capacitive resonators where each electrode adds a negative electrical stiffness to the resonator, reducing its resonant frequency.

\subsubsection{Electromechanical Modeling}

Similar to mechanical coupling where the resonators are mechanically connected with compliant mechanical elements, the electrostatically coupled beam resonators are coupled by an electrostatic negative stiffness distributed along their length. In order to develop a lumped-element mechanical equivalent model for the electrostatically coupled beam filter, the overall effective contribution of all the partial stiffness elements between the beams should be calculated.

As it was mentioned in the previous chapter, the effective mechanical stiffness of a beam resonator is a function of the position along the beam length: 


$$
K_{\text {mech.eff }}(x)=\frac{K_{\text {mech.mid }}}{Y(x)^{2}}
$$

where $K_{\text {mech.eff }}(x)$ is the effective mechanical stiffness at position $x, K_{\text {mech.mid }}$ is the mechanical stiffness of the beam in its central position, and $Y(x)$ is the normalized mode shape function for the first flexural mode of a clamped-clamped beam resonator.

The degree to which each element of the electrostatic stiffness can contribute to the frequency response depends inversely on the effective mechanical stiffness at the location of the element. The larger the effective mechanical stiffness is, the weaker the effect of electrostatic stiffness on the resonator frequency is at that location. Therefore:

$$
d K_{\text {elec.eff }}(x)=Y(x)^{2} d K_{\text {elec }}
$$

In order to define an overall effective electrostatic stiffness with respect to the central position in the beam, the effective electrical stiffness at all points along the beam should be integrated:

$$
K_{\text {elec.eff }}=\int_{0}^{L} Y(x)^{2} d K_{\text {elec }}=-\frac{\varepsilon h_{r}\left(\Delta V_{p}\right)^{2}}{g_{c}{ }^{2}} \int_{0}^{L} Y(x)^{2} d x
$$

For clamped-clamped beam resonators resonating in their first flexural mode, the normalized mode shape function is [34]:

$$
Y(x)=\frac{\cosh \frac{\lambda_{1} x}{L}-\cos \frac{\lambda_{1} x}{L}-\sigma_{1}\left(\sinh \frac{\lambda_{1} x}{L}-\sin \frac{\lambda_{1} x}{L}\right)}{1.588}
$$


where $\lambda_{l}=4.73004074$ and $\sigma_{l}=0.982502215$. Integrating the square of the normalized mode shape along the beam length using numerical methods results in $\int_{0}^{L} Y(x)^{2} d x=0.39 L$, therefore:

$$
K_{\text {elec.eff }}=-\frac{0.39 \varepsilon h_{r}\left(\Delta V_{p}\right)^{2}}{g_{c}{ }^{3}}
$$
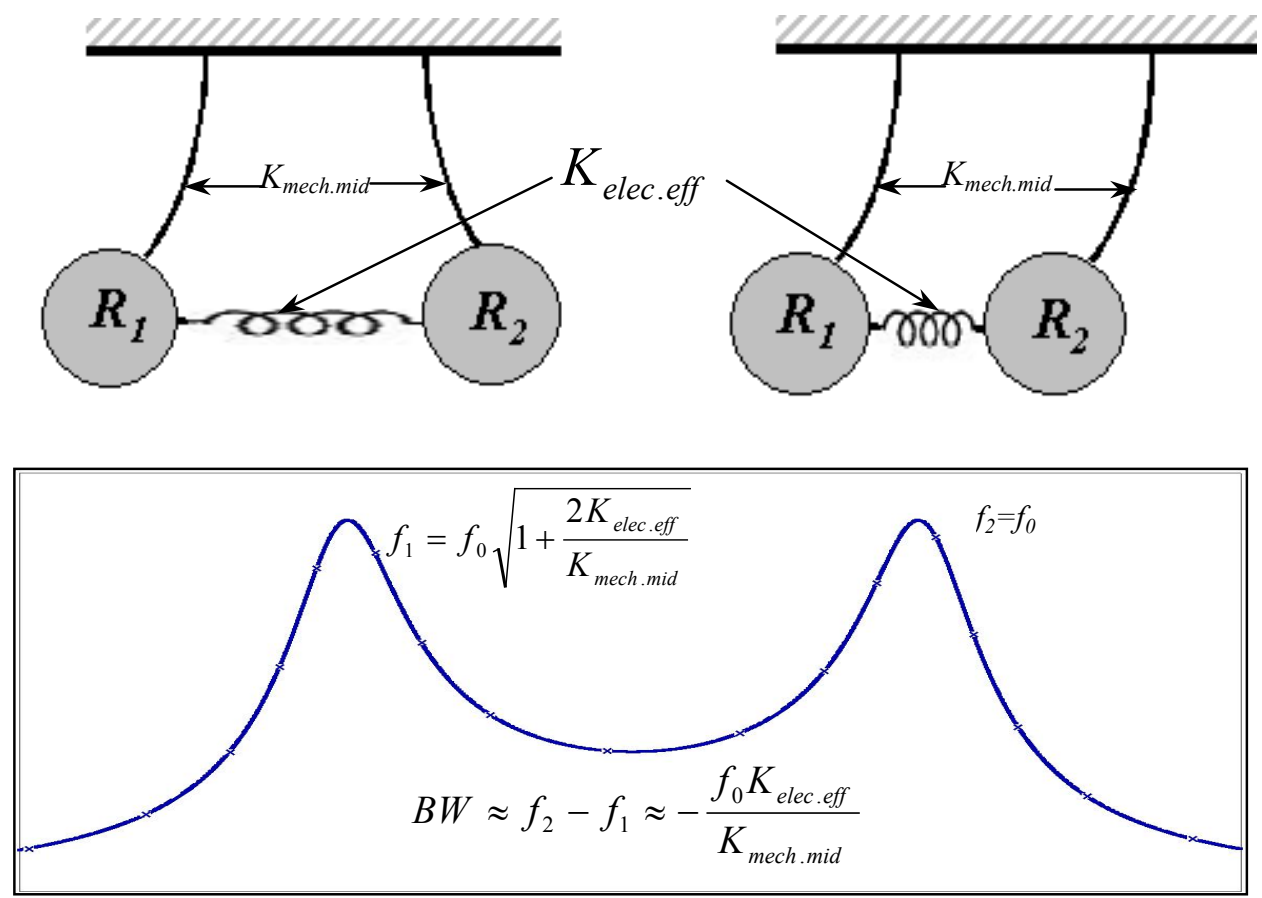

Figure 4.13. Equivalent lumped element model of a second order electrostatically coupled filter and its frequency response.

Figure 4.13 shows the mechanical equivalent lumped element schematic of a second order electrostatically coupled filter and its frequency response. Unlike the mechanically coupled filters where the resonators move in-phase in the first resonant mode and out-of-phase in the second resonant mode, the resonators are out-of-phase in 
the first mode of an electrostatically coupled filter, and vice versa. This stems from the fact that the electrostatic coupling spring has a negative effective value and reduces the stiffness of the resonator causing a lower frequency resonant mode when interfering with the resonators (in their out-of-phase motion).

In contrast to the mechanical coupling approach where the coupling strength and consequently the filter bandwidth is determined only by the stiffness and position of the coupling element, the coupling strength in electrostatically-coupled arrays is a function of the polarization voltages as well as the geometrical parameters, i.e., the coupling area and gap spacing. Therefore, characteristics of electrostatically coupled filter can be tuned/programmed by changing the polarization voltages to reach the target specifications.

To derive an electrical equivalent circuit for the electrostatically coupled beam filters, the following conversions from the mechanical to the electrical domain should be performed:

$$
\begin{gathered}
\text { velocity (v) } \rightarrow \text { current (1) } \\
\text { force (f) } \rightarrow \text { voltage (v) }
\end{gathered}
$$

so that stiffness is converted to the inverse of capacitance, mass to inductance, and damping to resistance. The resonator is then represented by a series RLC tank. The force applied by the coupling stiffness is determined by the sum of the displacements of the resonators. Therefore, the coupling element will be presented by a parallel negative capacitor to ground at the coupling node in between the two resonators so that the sum of the currents of the resonators will determine the voltage of the coupling capacitor. 
Based on the discussion above, the electrical equivalent circuit for a second order electrostatically coupled filter is as shown in Fig. 4.13. The value of the series capacitor of the resonators $\left(C_{i o}\right)$ depends on the mechanical properties of the resonators (mass and stiffness) as well as the electromechanical coupling strength between the input and output electrodes and the resonating body. The electromechanical coupling in turn depends on the applied polarization voltages and the capacitive gap between the electrodes and the resonators, as well as the electrode area (as derived in Chapter III). Therefore:

$$
C_{i o}=\frac{1}{R_{i o} Q \omega_{0}}=\frac{C_{s o} C_{d o} V_{p 1} V_{p 2}}{\sqrt{K_{\text {mech }} M} \omega_{0} g_{d} g_{s}}
$$

where, $R_{i o}$ is the equivalent resistance of the resonators, $Q$ is the mechanical quality factor of individual resonators, $C_{s o}$ and $C_{d o}$ are the sense and drive electrode capacitances respectively, $g_{d}$ and $g_{s}$ are the sense and drive capacitive gap sizes, $M$ is the equivalent mass of the resonators, and $\omega_{0}$ is the operating angular frequency of the resonators.

Since the capacitor values are inversely proportional to the stiffness that they represent, the value of the coupling capacitor will be:

$$
C_{c}=\frac{C_{i o} K_{\text {mech.mid }}}{K_{\text {elec.eff }}}
$$

The two terminating transformers count for any asymmetry between the input and output ports of the filter which results in different impedance levels at the input and output ports and their transformation coefficients are:

$$
T_{o i}=\frac{g_{s} A_{d} V_{p 1}}{g_{d} A_{s} V_{p 2}}=T_{i o}^{-1}
$$


Capacitors $C_{i}$ and $C_{o}$ at the input and output nodes, represent the physical pad and sense or drive capacitors existing at the input and output of the filter.

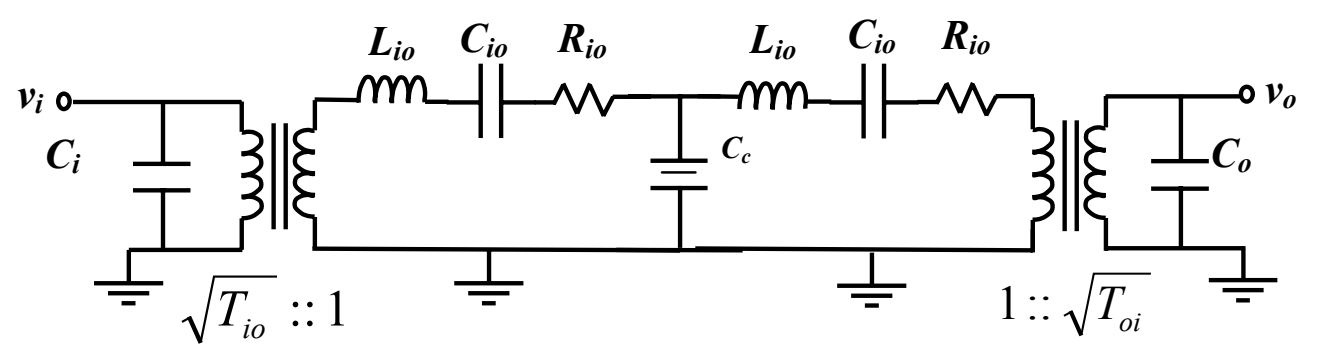

Figure 4.14. Electrical Equivalent circuit of a second order electrostatically coupled filter.

Due to similarity of the equivalent electrical circuit models for electrostatically and capacitively coupled filter arrays, the discussion on insertion loss for electrostatically coupled filters will be similar to the capacitively coupled arrays. 


\title{
CHAPTER V
}

\section{PRACTICAL DEMONSTRATION OF ELECTRICALLY COUPLED MICROMECHANICAL FILTERS}

\begin{abstract}
A variety of low frequency single crystal silicon (SCS) two-port capacitive beam resonators were used for practical demonstration of the electrically coupled micromechanical bandpass filters. Three different types of SCS resonators were used in this work: 1. Single crystal silicon beam resonators with polysilicon drive and sense electrodes fabricated through the HARPSS fabrication process [7,8]; 2. Fully SCS resonators consisting of SCS resonating element and SCS drive and sense electrodes separated by deep submicron dry etched capacitive gaps on SOI substrates [27]; and 3. Fully SCS resonators with lithography defined capacitive gaps fabricated on SOI substrates [33].
\end{abstract}

\subsection{Electrically Cascaded Filters}

Single crystal silicon beam resonators with polysilicon drive and sense electrodes fabricated through the HARPSS fabrication process were used for implementation of active coupled micromechanical filters. Fig. 5.1 shows the SEM view of the $300 \mu \mathrm{m}$ long, $6.5 \mu \mathrm{m}$ wide, $25 \mu \mathrm{m}$ thick clamped-clamped SCS beam resonator with a gap spacing of $700 \mathrm{~nm}$ used in this work. The individual resonators have a quality factor of $\sim 10,000$ at the center frequency of $600 \mathrm{kHz}$. 


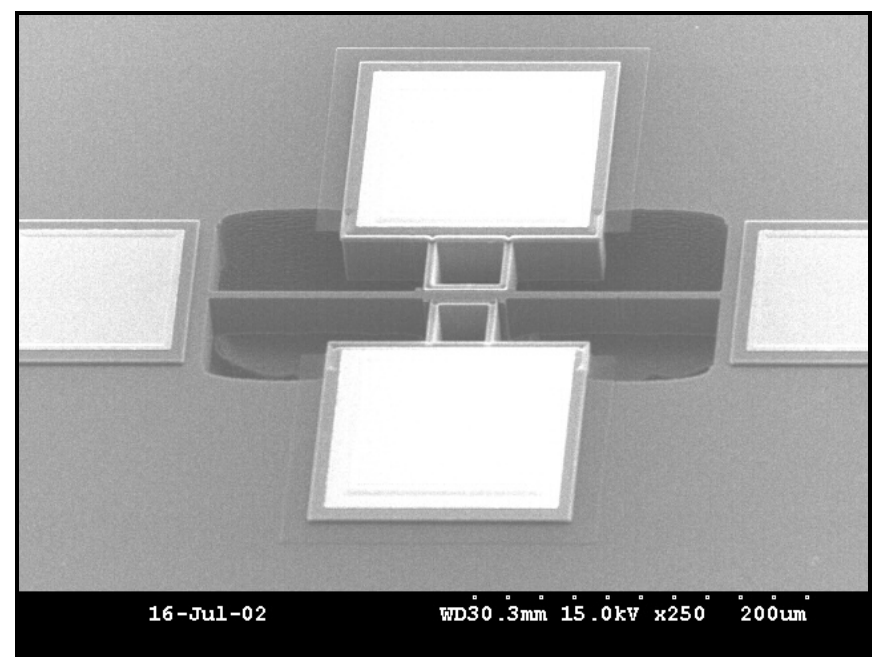

Figure 5.1. A $300 \mu \mathrm{m}$ long, $6.5 \mu \mathrm{m}$ wide, $25 \mu \mathrm{m}$ thick clamped-clamped beam HARPSS SCS resonator with a gap spacing of 700nm.

The resonators were mounted and wire-bonded on a PCB containing up to four amplifier channels to sense the output signal of the resonators. The PCB was placed in a custom vacuum system, which kept the pressure below 1mTorr. Wideband, low noise, JFET-input operational amplifiers (op-656) were used in a non-inverting configuration for implementation of the amplifiers. For active coupling of the resonators, the output of each resonator was connected to an amplifier and the output signal of the amplifier was applied to the drive electrode of the resonator of the next stage. Figure 5.2 shows the schematic diagram of the test setup. Different polarization voltages $\left(V_{p}\right)$ provided by the DC power supply (1-40V) were applied to the substrates that are electrically connected to the body of the resonators. A small AC signal $\left(\sim 1 \mathrm{mV}_{\mathrm{p}-\mathrm{p}}\right)$ provided by the network analyzer was applied to the drive electrode of the first resonator. The output signal from the sense electrode of the second resonator is detected and amplified by the interface circuit and sent back to the network analyzer. The frequency of the applied signal sweeps over a frequency range covering the passband of the filter and the frequency response is 
measured and displayed by the network analyzer. An Agilent 4395A network analyzer was used in this work.

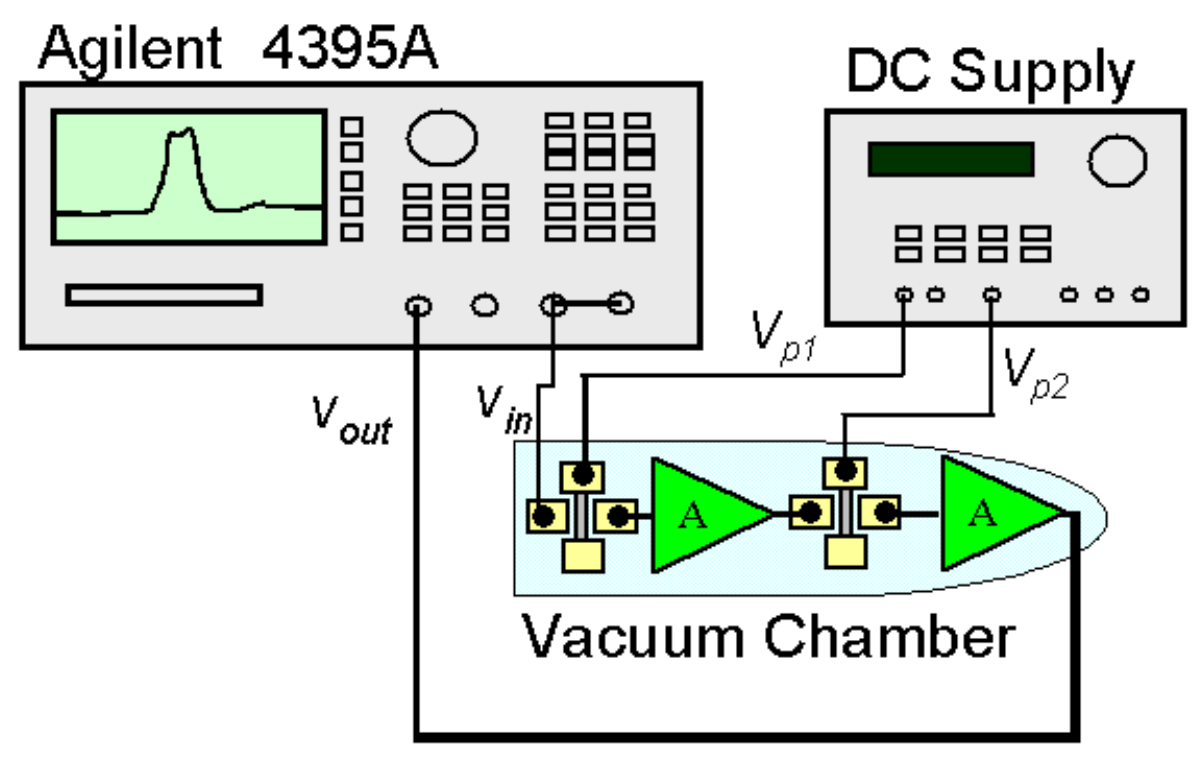

Figure. 5.2. Schematic diagram of the test setup used for demonstration of second and third order electrically cascaded micromechanical filters.

Frequency responses of second and third order cascaded stages were measured using the described test setup. Fig. 5.3 shows the frequency response of up to 3 stages of cascaded resonators with equal center frequencies $(600 \mathrm{kHz})$. An overall Q of 19,300 (about 2X Q amplification) was achieved by cascading three resonators with individual Q of $\sim 10,000$. It is also demonstrated that the $20 \mathrm{~dB}$ shape factor is reduced from 10.7 to 3.6, by increasing the number of stages from one to three. Table 5.1 summarizes the measurement results and analytically calculated values of overall Q achieved via active cascading of the resonators at different center frequencies showing excellent agreement between the measured and calculated values. 


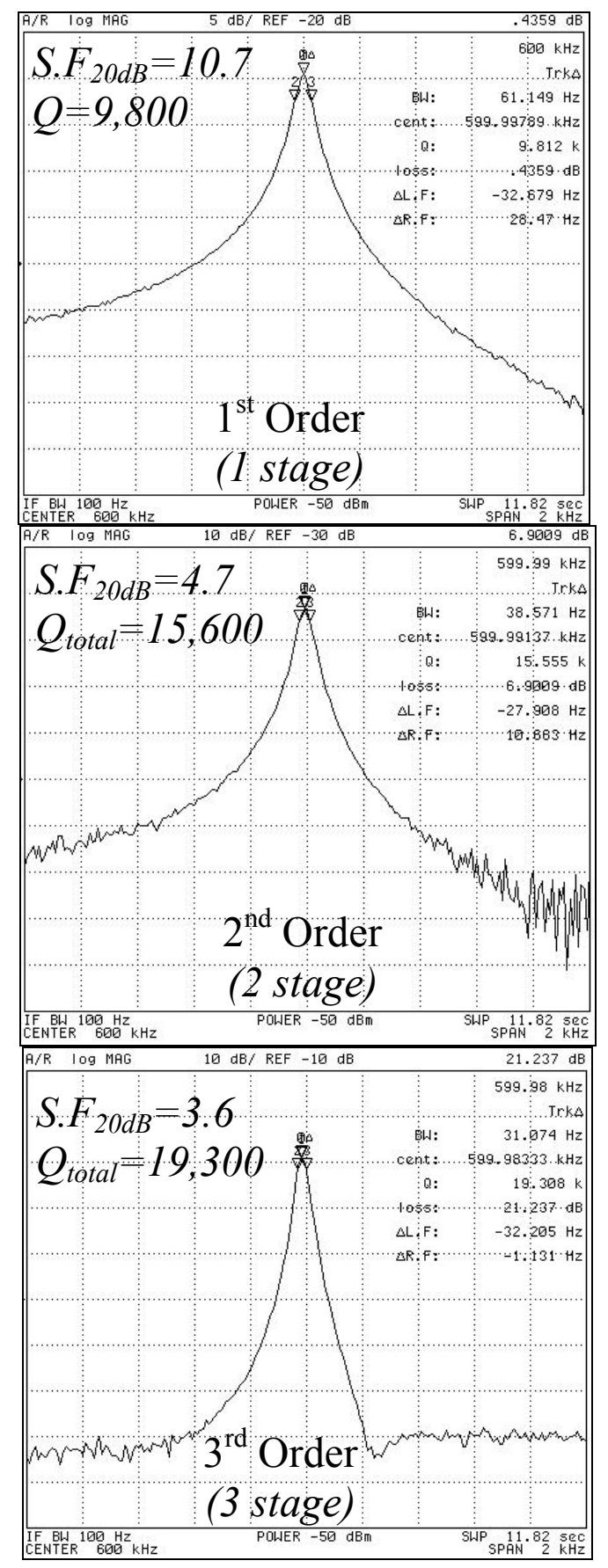

Figure 5.3. Measured frequency response of cascaded resonators demonstrating $Q$ amplification and shape factor improvement by increasing the number of stages. 
Table 5.1. Calculated and measured overall and individual quality factors for cascaded resonators.

\begin{tabular}{|c|c|c|c|c|c|}
\hline$f_{0}$ & $\begin{array}{c}Q_{1} \\
1^{\text {st }} \text { stg }\end{array}$ & $\begin{array}{c}\mathbf{Q}_{2} \\
2^{\text {nd }} \text { stg }\end{array}$ & $\begin{array}{c}Q_{3} \\
3^{\text {rd }} \text { stg }\end{array}$ & $\begin{array}{c}Q_{\text {cascade }} \\
\text { Theory }\end{array}$ & $\begin{array}{c}Q_{\text {cascade }} \\
\text { Measured }\end{array}$ \\
\hline $60.5 \mathrm{kHz}$ & 17,500 & 16,300 & - & 26,300 & 28,100 \\
\hline $449 \mathrm{kHz}$ & 10,000 & 18,900 & - & 22,900 & 23,800 \\
\hline $599 \mathrm{kHz}$ & 9,800 & 10,600 & - & 15,900 & 15,600 \\
\hline $599 \mathrm{kHz}$ & 9,800 & 10,600 & 9,000 & 19,100 & 19,300 \\
\hline
\end{tabular}

In order to achieve larger bandwidth, center frequency of the cascaded stages were tuned to slightly different frequencies by adjusting the polarization voltages. Fig. 5.4 illustrates the frequency responses of the second and third order filters in this case. Due to active nature of the coupling technique, passband gain of larger than unity can be achieved.

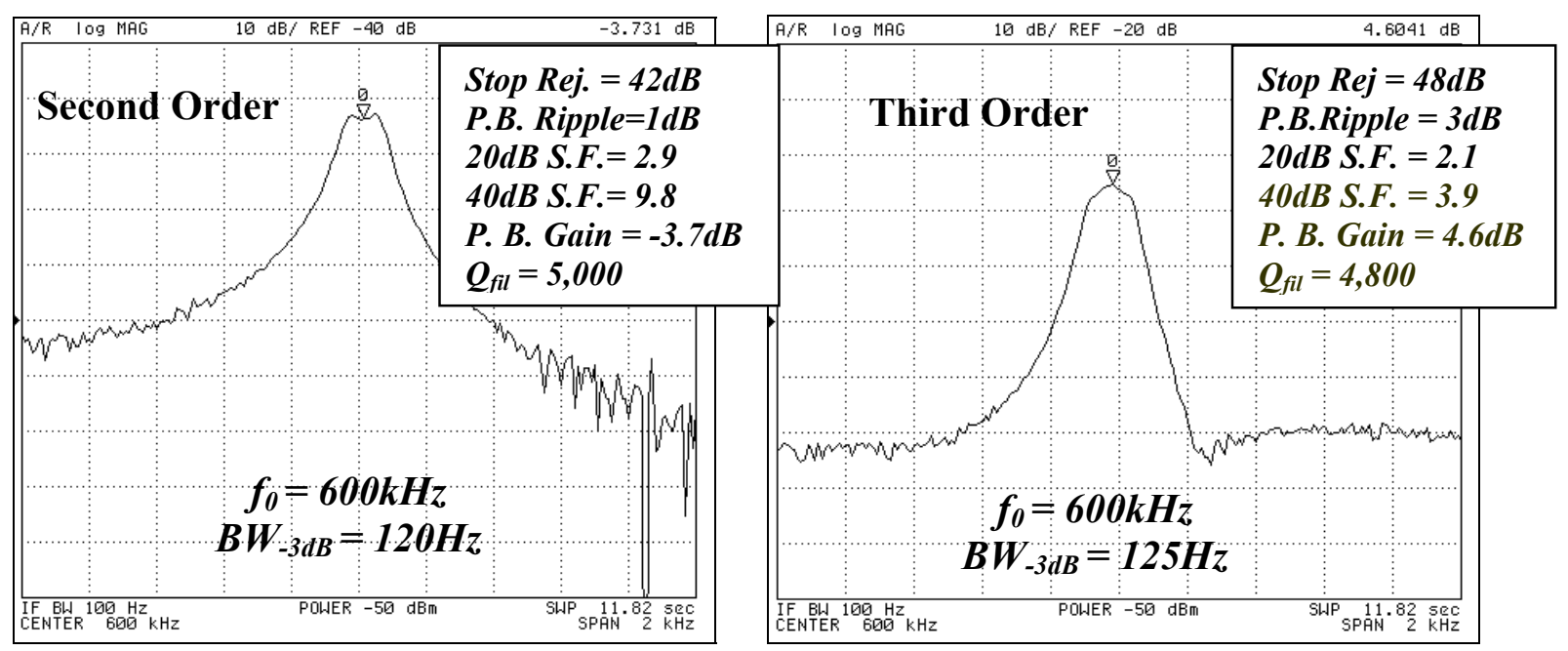

Figure 5.4. Frequency response of $2^{\text {nd }}$ and $3^{\text {rd }}$ order bandpass filters achieved by active electrical cascading of HARPSS resonators. 


\subsection{Discrete Capacitively Coupled Filters}

Similar test setup was used for demonstration of capacitive coupling using HARPSS capacitive SCS resonators. However, the output port of the first resonator was directly connected to the second resonator through wirebonds and the metal track on the PCB. The coupling node (output of the first stage) was connected to DC ground via a large resistor $(2 \mathrm{M} \Omega)$ to set a zero DC bias voltage at the coupling node while having negligible effect on the AC performance of the system. The schematic of the test setup is shown in Fig 5.5. Different polarization voltages $\left(V_{p}\right)$ were applied to the two resonators for frequency tuning.

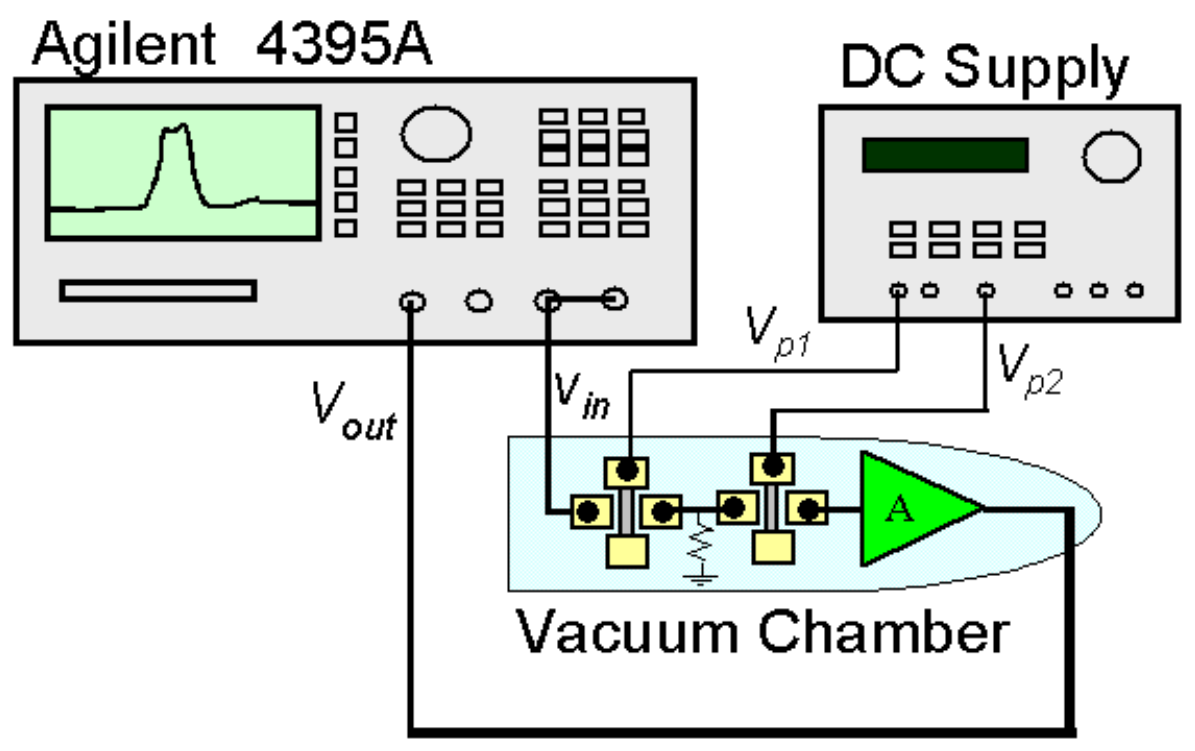

Figure 5.5. Schematic of the test setup for arrays of capacitively coupled SCS beam resonators.

Center frequency of the resonators were tuned to equal frequencies by adjusting the applied polarization voltages. No coupling capacitor was added and the parasitic capacitors contributing in the test setup (i.e. pad capacitance of the resonators and 
capacitance incorporated by the wirebonds, metal tracks on the PCB and the biasing resistor) having an overall value of $\sim 3 \mathrm{pF}$, was used as the coupling capacitor. Due to the large value of the coupling capacitor introduced by the parasitics comparing to the typical coupling capacitor values required, separation of the two resonance peaks is less than the bandwidth of the individual resonators (Fig. 5.6a) and two separate resonance peaks are not visible in the frequency response. However measured $20 \mathrm{~dB}$ shape factor of 3.0 which is much smaller than the calculated $20 \mathrm{~dB}$ shape factor for a first order filter ( 9.97 calculated using Eq. 4.2), confirms the second order nature of the resonance. PSPICE simulation of the system including the parasitic capacitors, shows excellent agreement with the measured resonance characteristics (Fig. 5.6b).

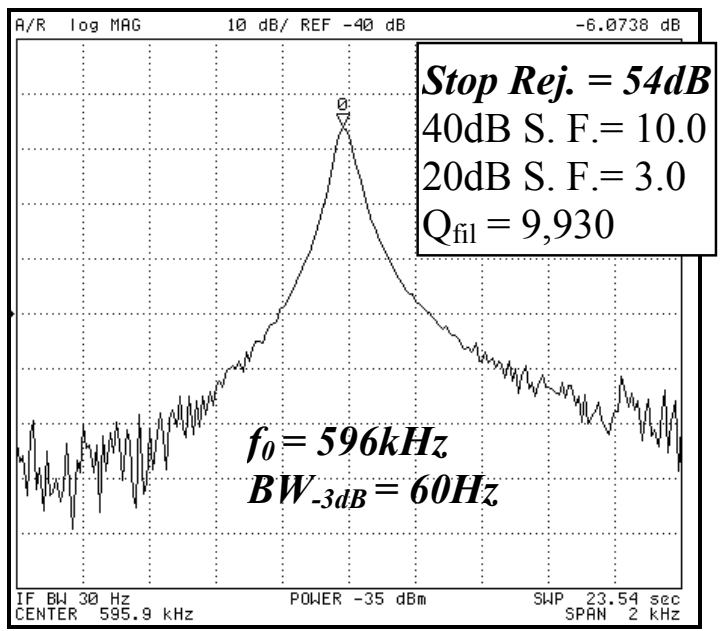

(a)

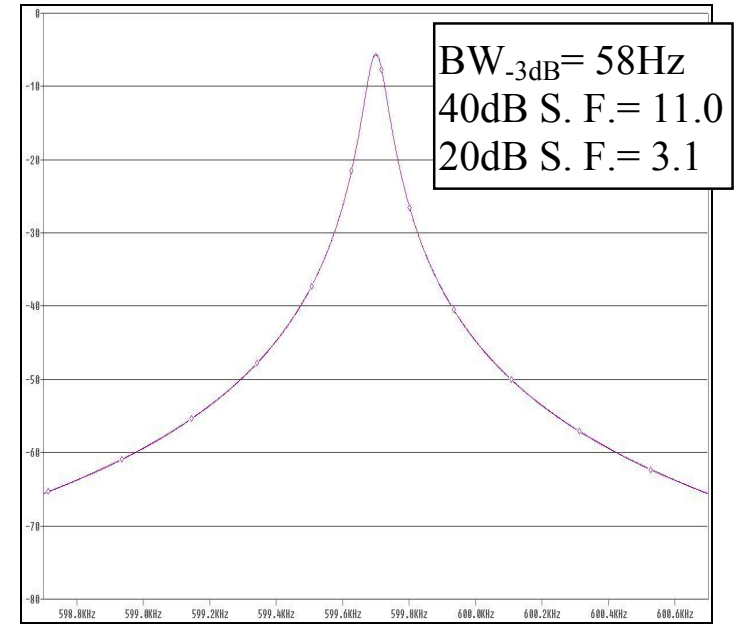

(b)

Figure 5.6. Frequency response of capacitively coupled resonators with off-chip interconnections a) measured frequency spectrum; b) PSPICE Simulation result.

To obtain a larger bandwidth, the two resonance peaks were separated by adjusting the applied DC polarization voltages and introducing a slight frequency mismatch between the resonators, which resulted a signal attenuation comparing to the 
previous case (Fig 5.6) as shown in Fig. 5.7a. The PSPICE simulation result presented in Fig. 5.7b shows excellent agreement with the measured frequency response.

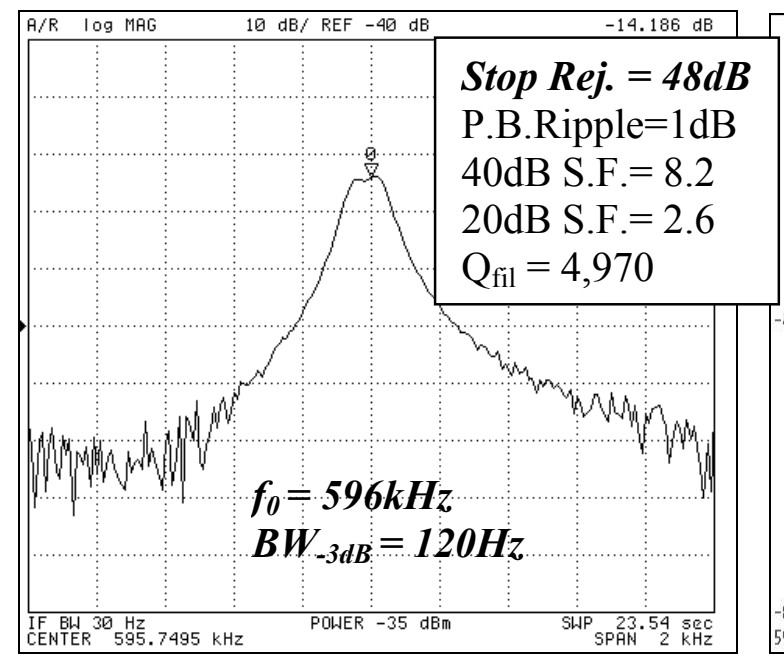

(a)

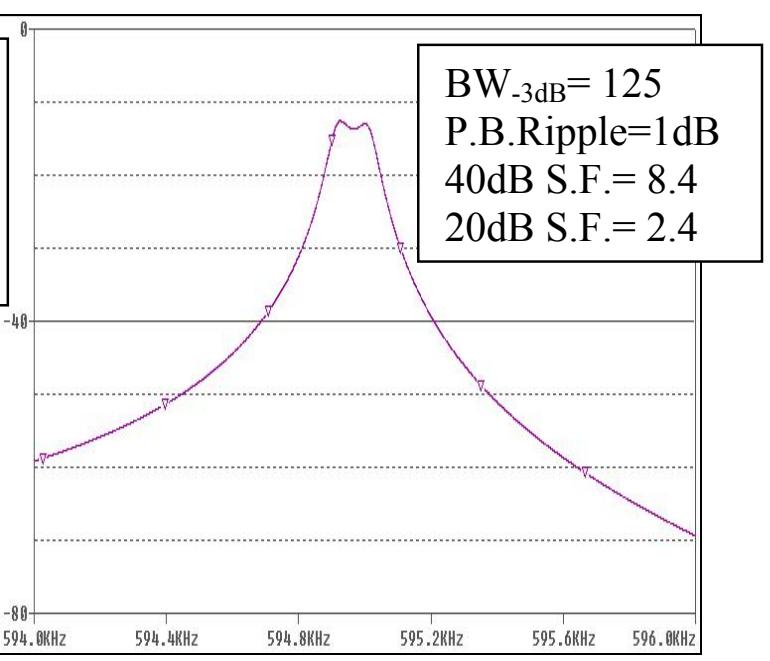

(b)

Figure 5.7. Frequency response of the capacitively coupled resonators after adjusting the polarization voltages to increase the bandwidth a) measured frequency response; b) PSPICE simulation result.

\subsection{Capacitively Coupled Filters with Integrated Coupling Capacitors}

Low frequency prototypes of capacitively coupled filters with integrated coupling capacitors were fabricated on SOI substrates using the nano-trench dry-etching technique [27]. Figure 5.8 shows the SEM view of a fabricated second order capacitively coupled bandpass filter. The filter consists of two clamped-clamped beam resonators each $178 \mu \mathrm{m}$ long, $3.7 \mu \mathrm{m}$ wide, and $4.5 \mu \mathrm{m}$ thick with $350 \mathrm{~nm}$ wide dry-etched capacitive transducer gaps. The quality factor of the individual resonators was measured to be 5,000 at resonance frequency of $800 \mathrm{kHz}$. The output electrode of the first resonator is connected to the input of the second resonator through a $50 \mu \mathrm{m}$ wide, $155 \mu \mathrm{m}$ long silicon island. 
The handle layer of the SOI substrate is connected to DC ground, so that the capacitance between the interconnecting silicon island and the handle layer counts as the coupling capacitor. The thickness of the buried oxide layer of the SOI substrate for the $800 \mathrm{kHz}$ filter of Fig. 5.8 is $5 \mu \mathrm{m}$ resulting in a $45 \mathrm{fF}$ coupling capacitor value.

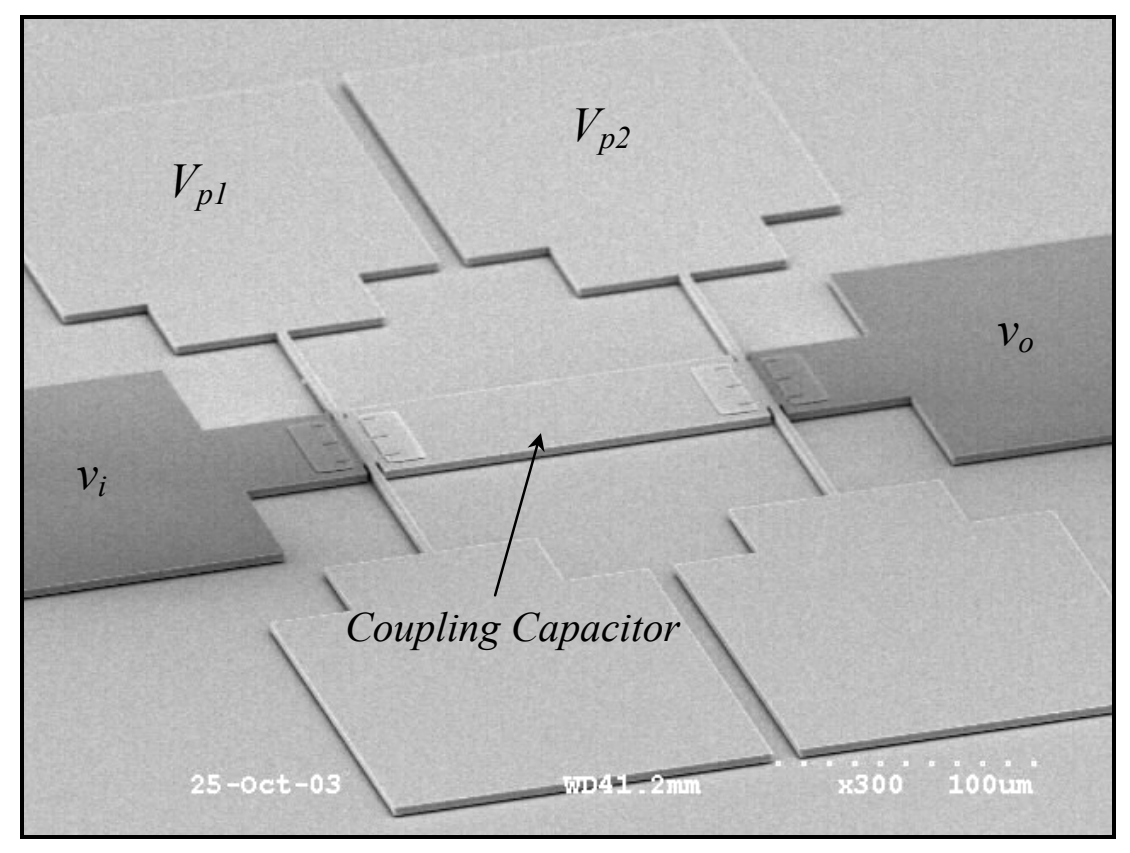

Figure 5.8. SEM view of a second order capacitively coupled filter with integrated coupling capacitor operating at $800 \mathrm{kHz}$. 


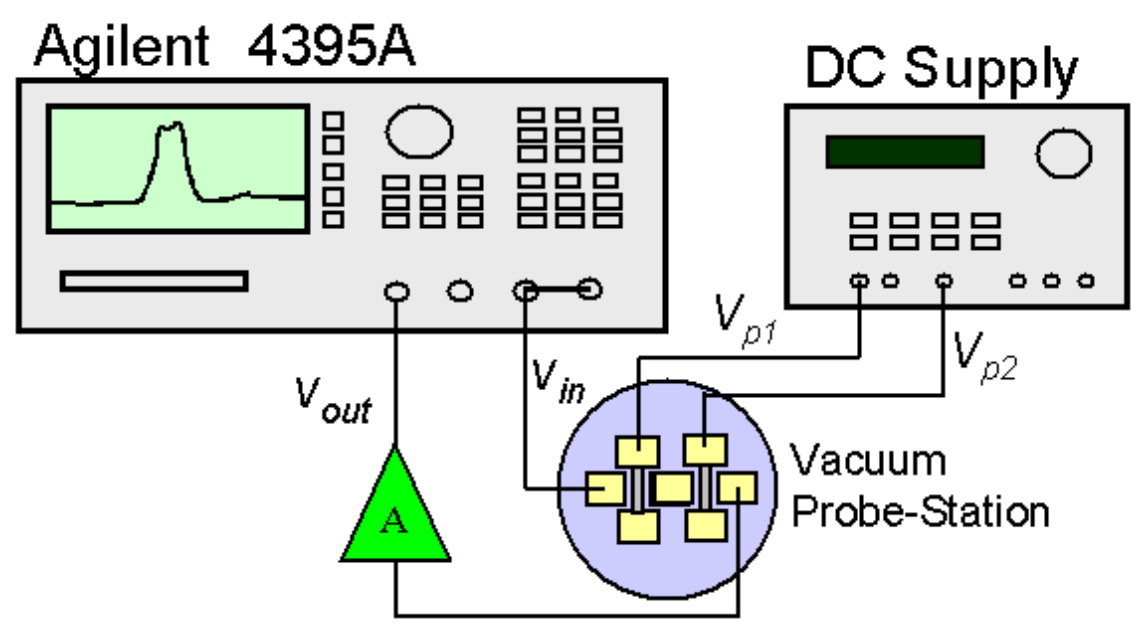

Figure 5.9. Schematic of the test setup for measurement of arrays of capacitively coupled SCS beam resonators with integrated coupling capacitor.

Figure 5.9 shows the schematic diagram of the measurement setup for integrated capacitively coupled filters. One of the main concerns for measurement of such devices was DC biasing of the coupling capacitor. Since very small values of coupling capacitors in the order of tens of femto-Farad are required for proper operation of such filters, addition of any extra biasing elements to set a constant DC bias value for the coupling capacitor, will cause a large overall capacitance at the coupling node. In order to alleviate the biasing problem without adding extra biasing elements, polarization voltages with opposite polarities were applied to the two resonators causing the coupling capacitor to have a DC value equal (or close) to the average of the two polarization voltages which will be close to zero. As a result there will be enough DC voltage difference for signal transduction between each of the resonators and the coupling capacitor that also acts as the input and output electrode of the coupled resonators.

Figure 5.10, shows the frequency response of the $800 \mathrm{kHz}$ filter of Fig. 5.8 with different values of polarization voltages applied to the resonators. As shown by the model derived in the previous chapter (Eq. 4.1), as the polarization voltages increase, the 
equivalent electrical impedance of the resonators decrease and consequently with the same value of coupling capacitor, stronger coupling (larger peak separation, and larger bandwidth) between the two resonators will occur. As shown in Fig. 5.10, the polarization voltages applied to the two resonators have different absolute values in order to compensate for fabrication non-idealities and tune the center frequency of the coupled resonators to equal values.
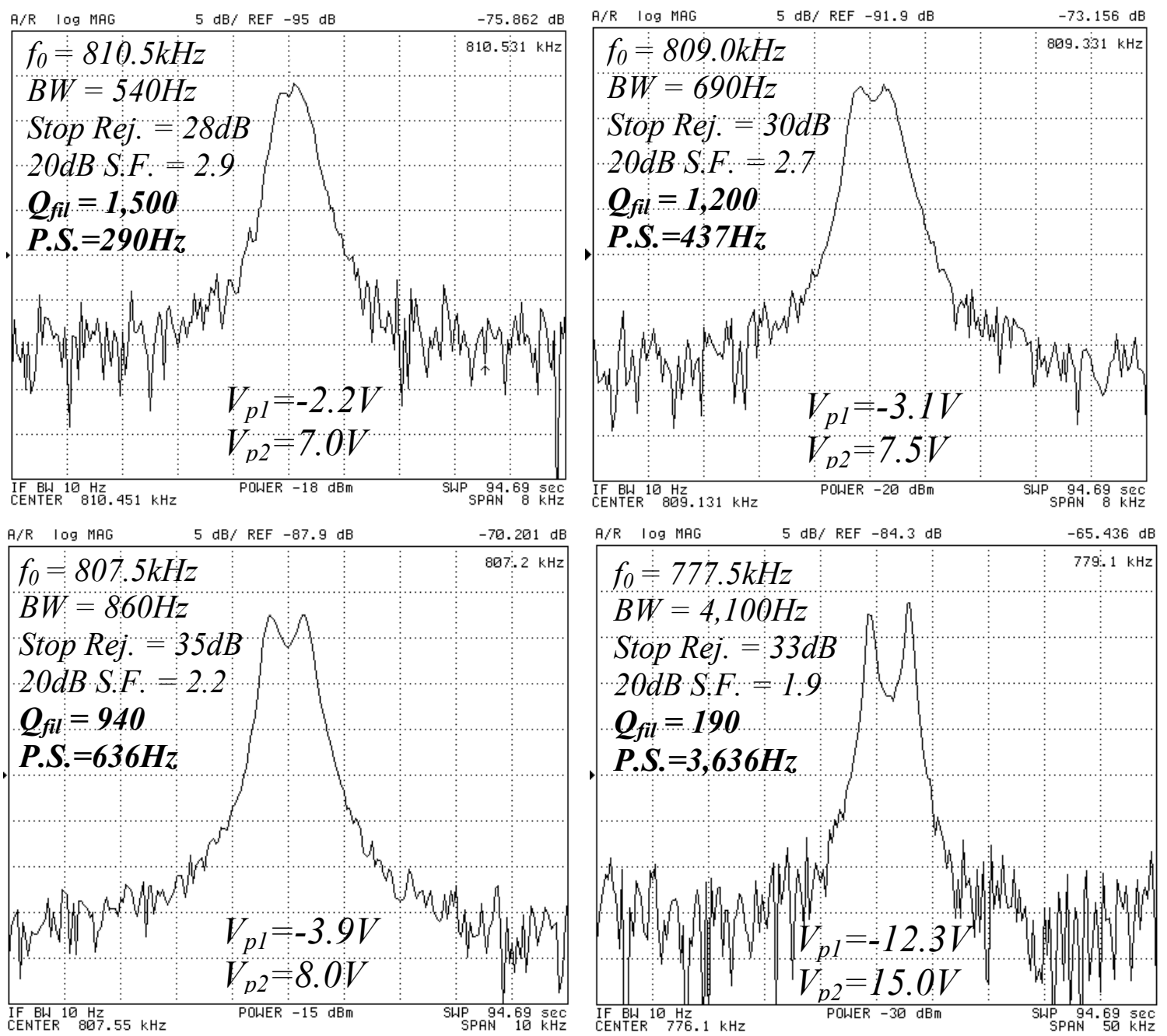

Figure 5.10. Frequency response of the $800 \mathrm{kHz}$ capacitively coupled filter with integrated coupling capacitor. Filter bandwidth changes due to the applied polarization voltages. 
Figure 5.11 shows the PSPICE simulation results using the equivalent circuit model presented in Chapter IV for the filter. Table 5.2, shows the theoretically calculated resonator parameters used in PSPICE simulation. Calculated separation of the resonance peaks using Eq. 4.1 for different values of polarization voltages match perfectly with the PSPICE simulation results and are presented in Table 5.2. For calculation of the equivalent motional resistance of the resonators, it has been assumed that the polarization voltages are divided equally between the coupling capacitor and the resonators and the motional resistances are the values experienced by the coupling capacitor at the coupling node with $V_{p}=\left(V_{p 1}+V_{p 2}\right) / 2$. Measured peak separation values are also presented in Table 5.2 for comparison with the theoretical values. Deviation of the measured data from the calculated values for some of the data points is considered to be a result of uneven distribution of the polarization voltages between the resonators and the coupling capacitor at the coupling node due to leakage currents.

Unfortunately due to existence of large pad capacitors $(\sim 1 \mathrm{pF})$ at the input and output of the filter, flattening of the passband by adding terminating resistors and valid measurement of insertion loss was not possible. 

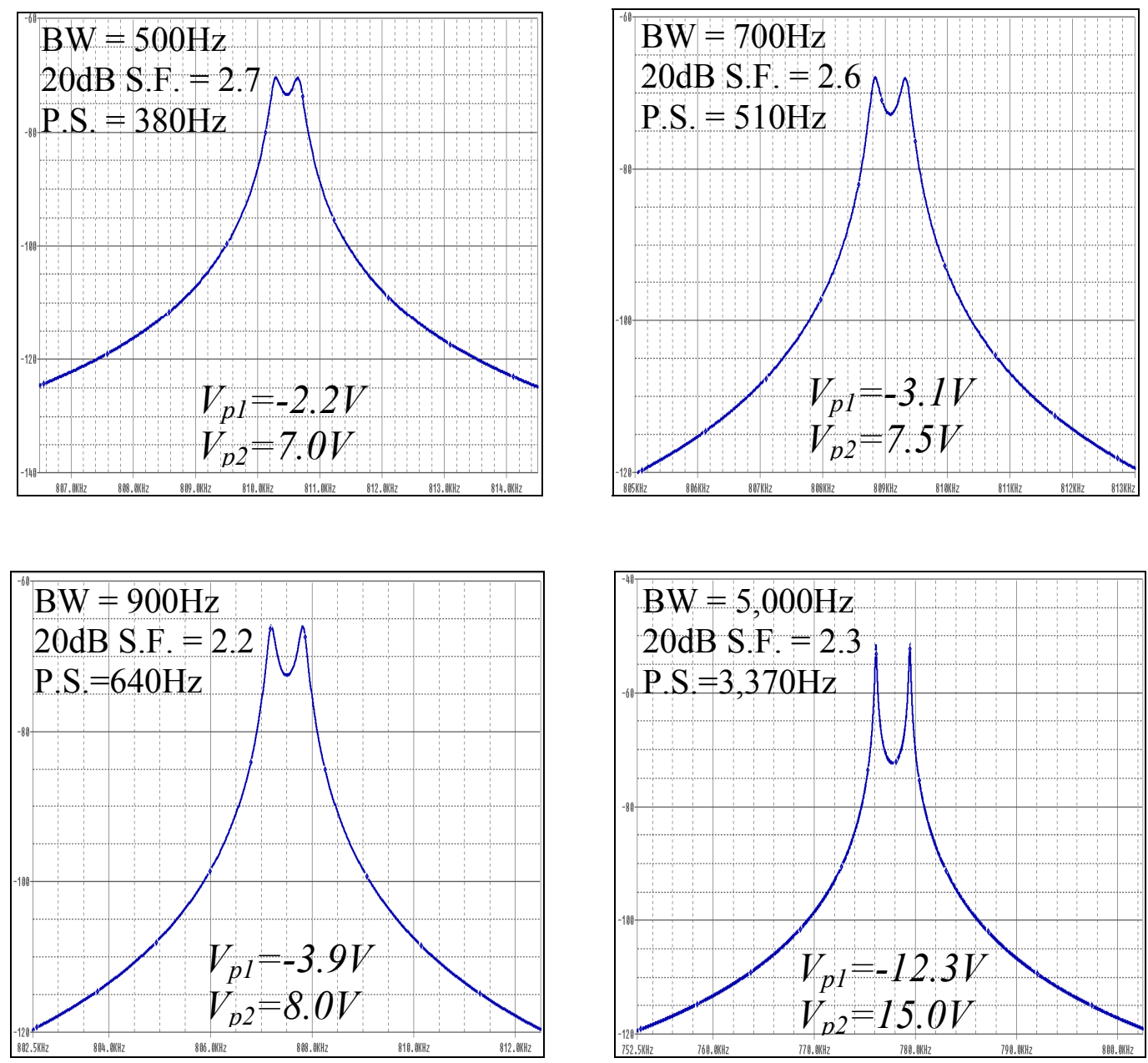

Figure 5.11. PSPICE simulation results for the capacitively coupled filter of Fig. 5.8 with polarization voltage values similar to the measurement conditions of Fig. 5.10.

Table 5.2. Calculated resonator parameters and filter bandwidth with different polarization voltages and the measured values.

\begin{tabular}{|c|c|c|c|c|}
\hline$V_{p 1}(V)$ & -2.2 & -3.1 & -3.9 & -12.3 \\
\hline$V_{p 2}(V)$ & 7.0 & 7.5 & 8.0 & 15.0 \\
\hline$R_{r e s}(M \Omega)$ & 1.84 & 1.39 & 1.1 & 0.21 \\
\hline $\begin{array}{c}\text { Calc. } \text { Simul. } \\
\text { P.S. }(\mathrm{Hz})\end{array}$ & 380 & 510 & 640 & 3,370 \\
\hline Meas. P.S. $(\mathrm{Hz})$ & 290 & 437 & 636 & 3,636 \\
\hline
\end{tabular}




\subsection{Electrostatically Coupled Filters}

For practical demonstration of the electrostatic coupling concept, single crystal silicon resonators with lithographically defined coupling and transducer gaps $(1-3 \mu \mathrm{m}$ wide) were fabricated on SOI substrates using a single mask fabrication process. The fabrication process consists of etching high aspect ratio trenches in the SOI device layer to the buried oxide followed by an undercut in HF. Figure 5.12 shows the SEM view of a fabricated second order beam filter. The filter is comprised of two $15 \mu \mathrm{m}$ thick, $5 \mu \mathrm{m}$ wide, $500 \mu \mathrm{m}$ long clamped-clamped beam resonators with a $2.8 \mu \mathrm{m}$ wide coupling gap in between.

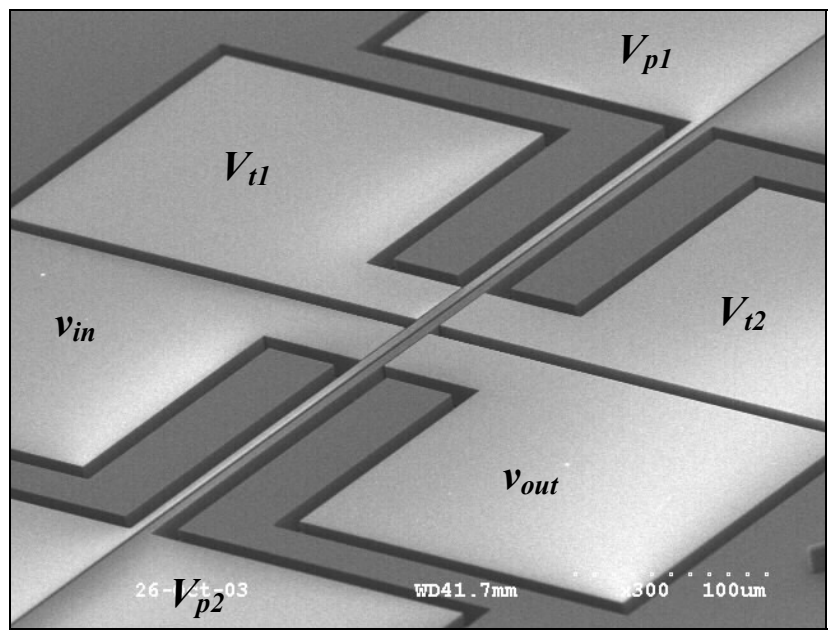

Figure 5.12. SEM of a fabricated second order clamped-clamped electrostatically coupled beam filter.

The fabricated filters were tested under vacuum in a custom vacuum chamber. As shown in Fig. 5.12, the sense and drive electrodes are split into two sections so that 
tuning voltages $\left(V_{t}\right)$ can be applied to one of the sections to adjust the resonant frequencies of the resonators to equal values while different polarization voltages are applied to them. However due to fabrication deficiencies and slight frequency mismatch between the coupled beams, the resonators can have equal center frequencies while different polarization voltages are applied to provide electrostatic coupling. In case of completely matched resonators and lack of split electrodes, application of equal polarization voltages with opposite polarities can provide the desired electrostatic coupling without the need for extra electrodes for tuning purposes.

Figure 5.13 shows the frequency responses exhibited by the filter of Fig. 5.12 with different sets of applied polarization voltages. Quality factor of individual resonators was measured to be 30,000 . As the polarization voltages increase, the frequency tuning slope increases and the required polarization voltage difference to compensate for the frequency mismatch of the beams will be smaller, resulting in a weaker coupling and smaller filter bandwidth. Resonance peak separations ranging from $202 \mathrm{~Hz}$ to $24 \mathrm{~Hz}$ (a bandwidth tunability of larger than one decade) was achieved for this filter by changing the polarization voltages (Fig. 5.13). Filter $\mathrm{Q}$ of $6800,40 \mathrm{~dB}$ shape factor of 12.5, and stopband rejection of higher than $50 \mathrm{~dB}$ has been achieved for the $24 \mathrm{~Hz}$ bandwidth filter of Fig. 5.13. Such high filter quality factors can not be easily achieved for the mechanically coupled filters unless ultra-compliant mechanical coupling elements are used. 

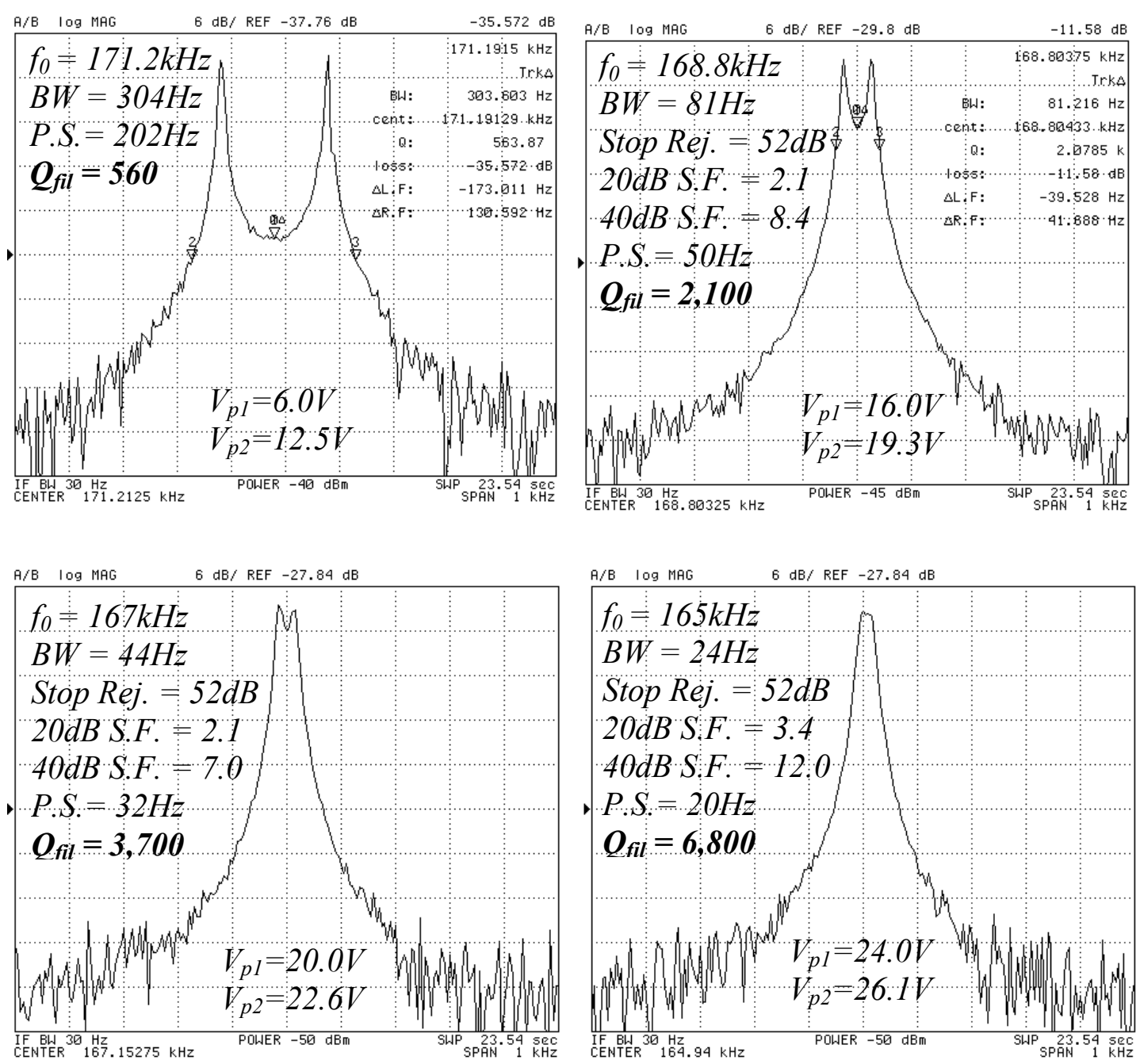

Figure 5.13. Frequency response of the electrostatically coupled filter of Fig. 5.12 with different sets of polarization voltages applied to the beams.

Due to existence of large pad capacitors at the input and output of the device, it was not possible to damp the resonators and flatten the passband by adding resistive terminations. For the same reason valid measurement of insertion loss was not possible.

Figure 5.14 shows the PSPICE simulation results for the tested filter under similar situations as shown in Fig. 5.13. The electrical equivalent circuit of Fig. 4.14 was used for the simulations and equivalent motional resistance, capacitance and inductance of the resonator as well as the coupling capacitor values are calculated using equations 4.16 and 
4.17. Table 5.3 contains the calculated element values used in PSPICE simulations as well as the resonance peak separations resulting from the simulation/calculation and the measured value. As shown in Table 5.3 there is an excellent agreement between the calculated, simulated and measured filter characteristics.
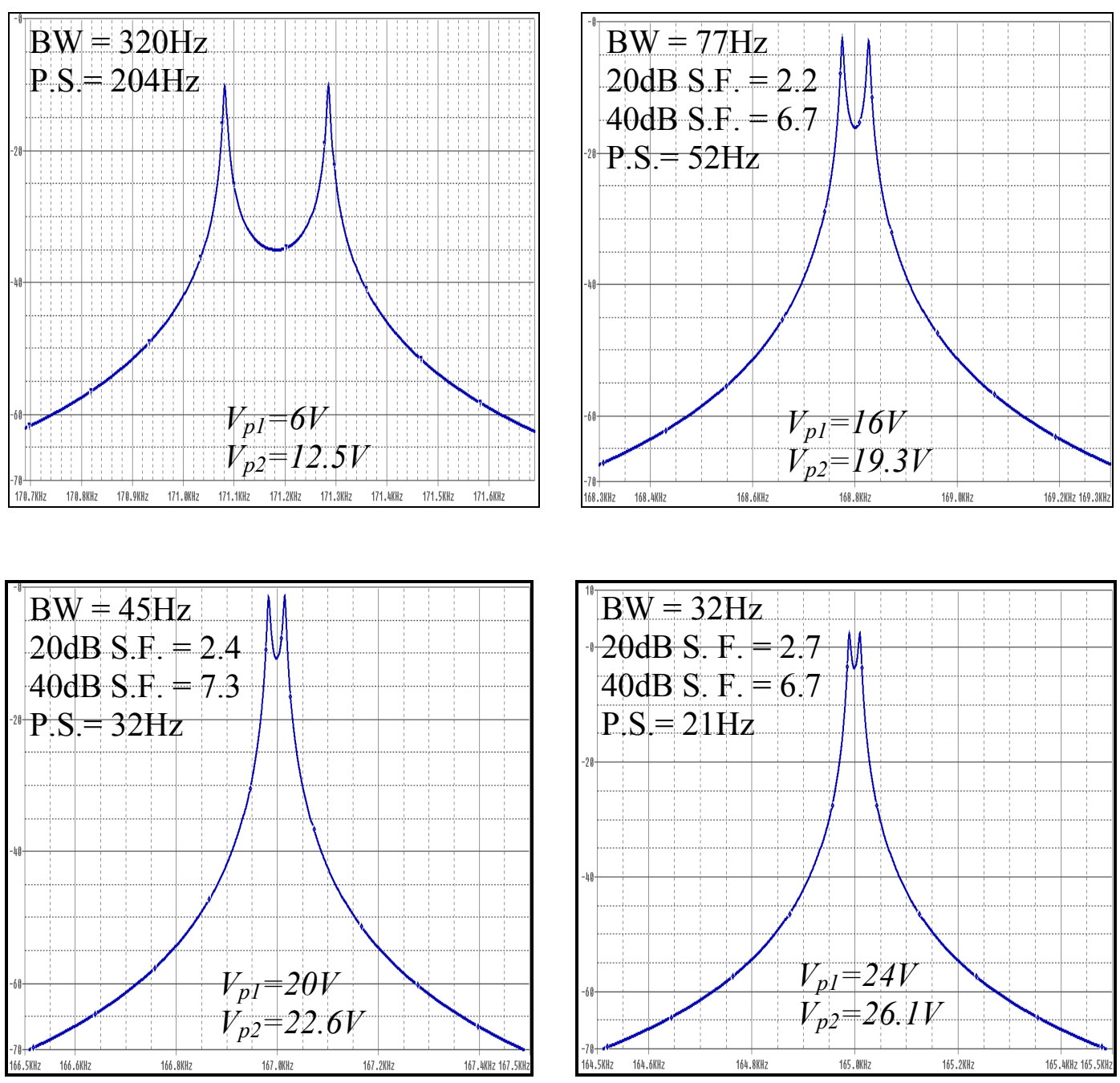

Figure 5.14. PSPICE simulation results for the electrostatically coupled filter of Fig. 5.12 with polarization voltage values similar to the measurement situation in Fig. 5.13. 
Table 5.3. Calculated resonator and coupling parameters used in PSPICE simulations of electrostatically coupled filter of Fig. 5.12 and comparison of simulated/calculated resonance peak separation with the measured values for different polarization voltages.

\begin{tabular}{|c|c|c|c|c|}
\hline$K_{r . m i d}(\mathrm{~N} / \mathrm{m})$ & \multicolumn{4}{|c|}{41.81} \\
\hline$M_{r . m i d}(\mathrm{ng})$ & \multicolumn{5}{|c|}{36.17} \\
\hline$R_{i o}(\mathrm{M} \Omega)$ & 3.1 & 0.753 & 0.514 & 0.371 \\
\hline$C_{i o}(a F)$ & 10.0 & 41.8 & 61.8 & 86.66 \\
\hline$L_{i o}(\mathrm{H})$ & 86500 & 21310 & 14710 & 10750 \\
\hline$K_{c}(\mathrm{~N} / \mathrm{m})$ & $-49.8 e-3$ & $-12.8 e-3$ & $-7.97 e-3$ & $-5.2 e-3$ \\
\hline$C_{c}(\mathrm{fF})$ & -8.4 & -136 & -324 & -697 \\
\hline$V_{p 1}(\mathrm{~V})$ & 6.0 & 16.0 & 20 & 24.0 \\
\hline$V_{p 2}(\mathrm{~V})$ & 12.5 & 19.3 & 22.6 & 26.1 \\
\hline $\begin{array}{c}\text { Calc. } / \text { Simul. } \\
\text { P.S }(\mathrm{Hz})\end{array}$ & 204 & 52 & 32 & 21 \\
\hline Meas. P.S. $(\mathrm{Hz})$ & 202 & 50 & 32 & 20 \\
\hline
\end{tabular}

As it was shown in Chapter IV:

$$
f_{2}-f_{1} \approx-\frac{f_{0} K_{\text {elec.eff }}}{K_{\text {mech.mid }}}
$$

Combining Eqs (4.15) and (5.1) results in:

$$
f_{2}-f_{1}=-\frac{0.39 \varepsilon L_{r} h_{r}\left(\Delta V_{p}\right)^{2}}{g_{c}{ }^{3} K_{\text {mech.mid }}} f_{0}
$$

showing that the frequency difference between the two resonance modes is a linear function of the polarization voltage difference squared $\left(\Delta V_{p}{ }^{2}\right)$ and the tuning slope is solely a function of the physical dimensions of the device. The calculated peak separation tuning slope for the $170 \mathrm{kHz}$ filter of Fig. 5 is $4.8 \mathrm{~Hz} / \mathrm{V}^{2}$. Measured values of peak separation for several values of polarization voltage difference as well as the theoretically predicted tuning characteristic with a slope of $4.8 \mathrm{~Hz} / \mathrm{V}^{2}$ are depicted in Fig. 5.15 showing excellent agreement between the theoretical trend and the measured data. 
Measured tuning slope resulting from linear extrapolation of the measured data points is $4.95 \mathrm{~Hz} / \mathrm{V}^{2}$.

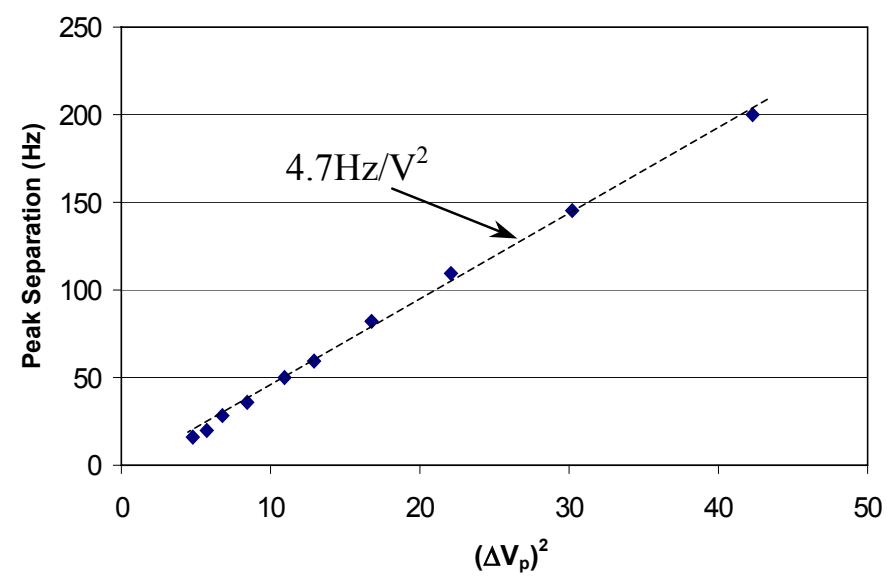

Figure 5.15. Theoretically calculated (dotted line) and measured data points (squares) showing separation of the two resonance peaks versus the square of polarization voltage difference $\left(\Delta \mathrm{V}_{\mathrm{p}}^{2}\right)$ for the electrostatically coupled filter of Fig. 5.12.

Electrostatically coupled filter exhibit even higher tunability comparing to the capacitively coupled arrays. For electrostatically filters the filter bandwidth can be sharply tuned by changing the polarization voltages. One can avoid change in the operating frequency and equivalent electrical impedance of the filter in this case by maintaining the polarization voltage difference between the electrodes and resonators. However for capacitively coupled filters, bandwidth tuning by changing the polarization voltages is always accompanied by shift in the impedance and operating frequency of the filter. 


\section{CHAPTER VI}

\section{CONCLUSION AND FUTURE DIRECTIONS}

The concept of electrical coupling of MEM resonators for implementation of higher order bandpass filters and its different variations was developed and discussed for the first time. Electrical coupling has higher potential for extension of the frequency into the UHF range comparing to the mechanical coupling approach and provides higher tunability for filters after fabrication.

Active coupling of micromechanical resonators using active amplifying elements in between the resonators was discussed and test results at $600 \mathrm{kHz}$ were demonstrated. Two times Q-amplification was achieved by cascading 3-stages of resonators with amplifying interconnects. Capacitive coupling of resonators using capacitors as the coupling elements with higher tuning and integration capability was discussed and demonstrated. Capacitively coupled second order bandpass filters with integrated coupling capacitors were implemented at operating frequency of $800 \mathrm{kHz}$. Finally, electrostatically coupled filters were presented in which no distinct coupling element is required and the electrostatic forces between closely-spaced resonators result in coupling between the resonators. Electrostatic coupling provides the highest tunability/programmability and filter characteristics can sharply be tuned by the applied polarization voltage values. Test results of a low frequency $(170 \mathrm{kHz})$ electrostatically coupled filter, shows over one decade of bandwidth tuning by changing the polarization voltages. 
Future work includes application of the presented electrical coupling techniques to ultra-stiff bulk mode SCS resonators [9] with operating frequencies in the HF (3$30 \mathrm{MHz})$, VHF $(30-300 \mathrm{MHz})$ and UHF $(0.3-3 \mathrm{GHz})$ band. Sub-100nm capacitive transducer gaps will be required for the resonators in order to provide low enough motional impedance for the resonators. For capacitively coupled filters the equivalent motional resistance of the resonators has to be scaled down proportionally to the frequency so that the same coupling capacitor values (in the order of tens of fF) can be used to provide a large enough coupling strength. The electrostatic coupling stiffness required for implementation of higher frequency electrostatically coupled filter arrays increases as the resonator stiffness increases. This calls for fabrication processes capable of producing submicron coupling gaps between the SCS structures. In addition, all types of microelectromechanical filters (mechanically or electrically coupled) require frequency tuning procedures for the individual resonators to have equal resonance frequencies for all the resonators in a filter array. This adds to the importance of having ultra-thin capacitive gaps in between the transducer or tuning electrodes and the resonating body of the resonators.

Fabrication and measurement techniques for cancellation or reduction of the input and output pad capacitors to allow proper resistive termination of the filters are also under investigation.

For active coupling of resonators, the performance can be dramatically improved by integration of the MEM resonators and CMOS electronics. Wide bandwidth, highly selective bandpass filters can be implemented using array architectures consisting of a number of parallel actively coupled resonator arrays as shown in Fig. 6.1. 


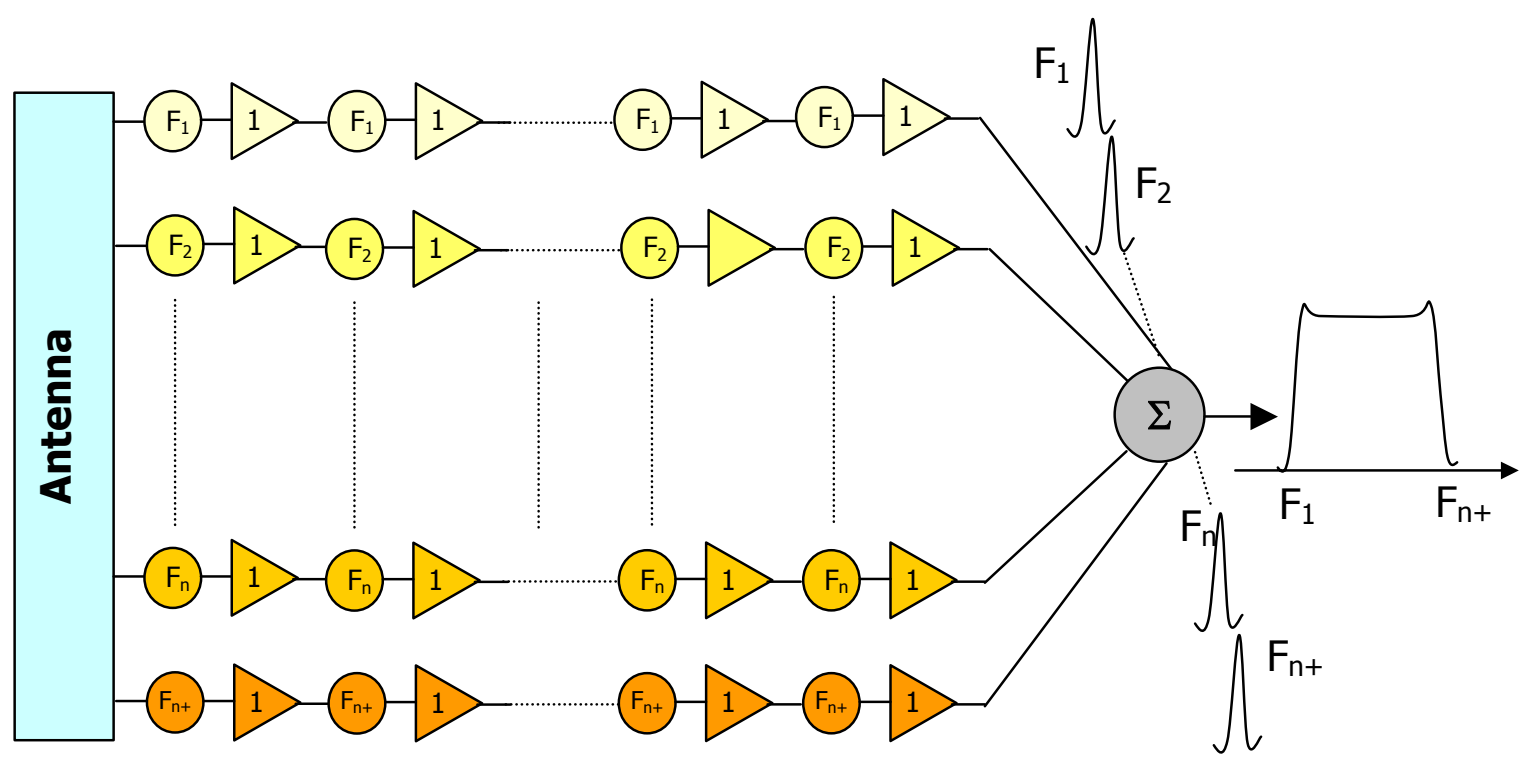

Figure 6.1. Schematic diagram of parallel actively coupled resonator arrays for implementation of wide bandwidth, highly selective MEM filters. 


\section{REFERENCES}

[1] M.W. Putty, S.C. Chang, R.T. Howe, A.L. Robinson, K.D. Wise, "One-Port Active Polysilicon Resonant Microstructures," Proceedings, An Investigation of Micro Structures, Sensors, Acutators, Machines, and Robots, IEEE, 1989, pp.60-65.

[2] R.T. Howe, "CMOS micromechanical resonator oscillator," Tech. Dig. IEEE Int. Electron Devices Meeting, Washington D.C., Dec. 5-8, 1993, pp.199-202.

[3] M. A. Abdelmoneum, et al, "Stemless wine-glass-mode disk micromechanical resonators", $M E M S^{\prime} 03$, pp. 698-701.

[4] W.-T. Hsu, J. Clark, C. T.-C. Nguyen, "Q-Optimized Lateral Free-Free Beam Micromechanical Resonators", Int. Conf. On Solid-State Sensors and Actuators (Transducer'01), Munich, Germany, 6/10-14/01, (2001), pp. 1110-1113.

[5] W.C. Tang, T.-C. Nguyen, and R.T. Howe, "Laterally driven polysilicon resonant microstructures", Sensors and Actuators, vol.20, pp. 25-32, 1989.

[6] J. R. Clark, W.-T. Hsu, and C. T.-C. Nguyen, "High-Q VHF micromechanical contour-mode disk resonators", IEEE Int. Electron Devices Meeting 2000, pp. 399-402.

[7] S. Y. No, et al, "Single crystal silicon HARPSS capacitive resonators with submicron gap spacings", proceedings, Hilton Head 2002, pp. 281-284.

[8] S. Pourkamali, et al, "High-Q Single Crystal Silicon HARPSS Capacitive Beam Resonators with Self-Aligned Sub-100nm Transduction Gaps", Journal of Micro Electro Mechanical Systems, August 2003, pp. 487-496.

[9] S. Pourkamali, and F. Ayazi, "SOI-based HF and VHF single-crystal silicon resonators with sub-100nm nanometer vertical capacitive gaps", Transducers '03, pp.837840 .

[10] T. Mattila et. al. "14MHz Micromechanical Oscillator", $11^{\text {th }}$ Int. Conf. on Solid-State Sensors and Actuators, (Transducer'01), Munich, Germany, 6/10-14/01, (2001), pp.1102-1105.

[11] V. Kaajakari et al, "Square-extentional mode single-crystal silicon micromechanical RF-resonator”, Transducers '03, pp. 951-954.

[12] R. A. Johnson, "Mechnical filters in electronics", A Wiley-interscience publication.

[13] F. D. Bannon, et al, "High frequency micromechanical IF filters", IEDM 96, pp. 773-776. 
[14] K. Wang, et al, "High-order medium frequency micromechanical electronic filters", JMEMS 8(4), 534, (1999).

[15] A.-C. Wong, et al, "Anneal-activated, tunable, $68 \mathrm{MHz}$ micromechanical filters", Sensors and Actuators 99, pp 1390-1393.

[16] L. Lin, et al, "Microelectromechanical Filters for Signal Processing", JMEMS 7(3), 286, (1998).

[17] D. S. Greywall, et al, "Coupled micromechanical drumhead resonators with practical applications as electromechanical. bandpass filters", J. Micromech. Microeng. 12 (2002), pp 925-938.

[18] K. Wang, et al, "Q-Enhancement of microelectromechanical filters via low velocity spring coupling", IEEE Ultrasonics symposium, 1997, pp. 323-327.

[19] H. C. Nathanson, W. E. Newell, R.A. Wickstrom and J.R. Davis, "The resonant gate transistor,” IEEE Trans. Electron Devices, vol. ED-14, pp. 117-133, 1967.

[20] M. E. Frerking, "Fifty years of progress in quartz frequency standards", 1996 IEEE international frequency control symposium, pp. 33-46.

[21] Y. L. Vorokhovsky, B.G. Drakhlis, "High-stability quartz oscillators on internally heated quartz resonators with AT and SC cuts", Proceedings of the 45th Annual Symposium on Frequency Control, pp. $447-451,1991$.

[22] V. T. Srikar, S. D. Senturia, "Thermoelastic damping in fine-grained polysilicon flexural beam resonators", JMEMS, Vol. 11, Issue: 5, Oct 2002, pp. 499-504.

[23] A. N. Cleland and M. L. Roukes, "Fabrication of high frequency nanometer scale mechanical resonators from bulk Si substrates", App. Phys. Lett., 69, 2653 (1996).

[24] F. Ayazi and K. Najafi, "High Aspect-Ratio Combined Poly and Single-Crystal Silicon (HARPSS) MEMS Technology”, JMEMS, 9(3), pp. 288-294, (2000).

[25] F. Ayazi and K. Najafi, "A HARPSS Polysilicon Vibrating Ring Gyroscope" in IEEE/ASME Journal of Microelectromechanical Systems, June 2001, pp. 169-179.

[26] A. Hashimura, "Single-crystal silicon HARPSS capacitive beam resonators", Master of science thesis, Department of Electrical and Computer Engineering, Georgia Institute of Technology, May 2002.

[27] S. Pourkamali, and F. Ayazi, "Fully single crystal silicon resonators with deepsubmicron dry-etched transducer gaps", MEMS'04, pp. 813-816.

[28] G. A. Campbell, "Physical theory of the electric wave-filter", Bell Syst. Tech. J., 1, pp. 1-32, Nov. 1922. 
[29] B. Jaffe, et al, "Piezoelectric Ceramics”, NewYork: Academic Press, 1971.

[30] S. Fujishima, et al, "Tuning fork resonators for electronic wrist watches using $\mathrm{ZnO}$ sputtered film", $1^{\text {st }}$ meeting on application of ferroelectric materials in Japan, 1977.

[31] J. H. Ginsberg, "Mechanical and structural vibrations", John Wiley \& Sons, Inc.

[32] S. Pourkamali, R. Abdolvand, and F. Ayazi, "A 600kHz Electrically Coupled MEMS Bandpass Filter,” MEMS‘03, pp. 702-705.

[33] S. Pourkamali, et al, "Electrostatically coupled micromechanical beam filters", MEMS'04, pp. 584-587.

[34] R.D. Blevins, Formulas for natural frequency and mode shape, New York: Van Nostrand Reinhold, 1979. 